\title{
Coherent Photon Manipulation in Interacting Atomic Ensembles
}

\author{
Callum R. Murray and Thomas Pohl \\ Max Planck Institute for the Physics of Complex Systems, Nöthnitzer Straße 38, 01187 Dresden, Germany \\ Department of Physics and Astronomy, Aarhus University, \\ Ny Munkegade 120, DK 8000 Aarhus C, Denmark \\ (Received 13 February 2017; revised manuscript received 21 June 2017; published 13 July 2017)
}

\begin{abstract}
Coupling photons to Rydberg excitations in a cold atomic gas yields unprecedentedly large optical nonlinearities at the level of individual light quanta. Here, the basic mechanism exploits the strong interactions between Rydberg atoms to block the formation of nearby dark-state polaritons. However, the dissipation associated with this mechanism ultimately limits the performance of many practical applications. In this work, we propose a new approach to strong photon interactions via a largely coherent mechanism at drastically suppressed photon losses. Rather than a polariton blockade, it is based on an interaction-induced conversion between distinct types of dark-state polaritons with different propagation characteristics. We outline a specific implementation of this approach and show that it permits us to turn a single photon into an effective mirror with a robust and continuously tunable reflection phase. We describe potential applications, including a detailed discussion of achievable operational fidelities.
\end{abstract}

DOI: 10.1103/PhysRevX.7.031007

\section{INTRODUCTION}

The notion that photons are devoid of mutual interactions in vacuum is well rooted in our elementary understanding of light. Nevertheless, the ability to engineer such interactions synthetically would hold profound implications for both fundamental and applied science, and has since ushered in a new era of research into nonlinear optics at the ultimate quantum level [1]. Intense efforts have been directed towards enhancing light-matter coupling through tight mode confinement [2-14] in order to achieve local nonlinearities by interfacing photons with a single quantum emitter. A complementary strategy, which is rapidly gaining momentum, exploits the collective coupling of light to particle ensembles with finite-range interactions [15-20] to establish large and nonlocal nonlinearities.

Interfacing light with strongly interacting atomic Rydberg ensembles [21-34] under conditions of electromagnetically induced transparency (EIT) [35] has emerged as a particularly promising way to implement this new type of mechanism $[15,16,36-45]$. EIT in these systems is based on the formation of Rydberg dark-state polaritons, which correspond to coherent superposition states of light and matter that are immune to dissipation $[46,47]$. While this polariton formation supports the lossless and form-stable propagation of single photons, the strong mutual interaction between two such polaritons

Published by the American Physical Society under the terms of the Creative Commons Attribution 4.0 International license. Further distribution of this work must maintain attribution to the author(s) and the published article's title, journal citation, and DOI.
Subject Areas: Atomic and Molecular Physics, Optics, Quantum Physics can easily perturb and break the underlying EIT condition, thereby rendering light propagation highly nonlinear [48-50]. Indeed, there have now been a number of experiments that demonstrated controllable photon-photon interactions of unprecedented strength in such systems [37,48-59].

Key to this nonlinearity is the destruction of EIT conditions that originates from an effective polariton blockade, whereby multiple proximate photons are prevented from simultaneously forming dark-state polaritons. As an immediate consequence, the emergent photon interactions inevitably carry an intrinsic dissipative component. Nevertheless, the nonlinear quantum optical response achieved in this way can be utilized to facilitate a broad range of applications, such as imaging [60,61], all-optical switches and transistors [54-57,62], quantum gates [42,58], and single-photon sources [43,48] and subtractors [59]. Yet, it turns out that high-fidelity operations require conditions (e.g., high atomic densities) where the performance of such applications is ultimately eclipsed by additional decoherence effects [54,62-64].

As a solution to this outstanding issue, we describe here a novel approach to quantum optical nonlinearities in a Rydberg-EIT medium without the polariton blockade. It exploits the atomic interactions to modify EIT conditions, rather than destroying them entirely. Generally, the devised strategy can thus be understood as a dark-state polariton switch, as opposed to the existing schemes based on the polariton blockade, Fig. 1(a). Consequently, this new mechanism globally preserves EIT conditions such that nonlinear dissipation is intrinsically suppressed, thereby alleviating the decoherence-related hindrances discussed in Refs. [62,63]. 
(a)

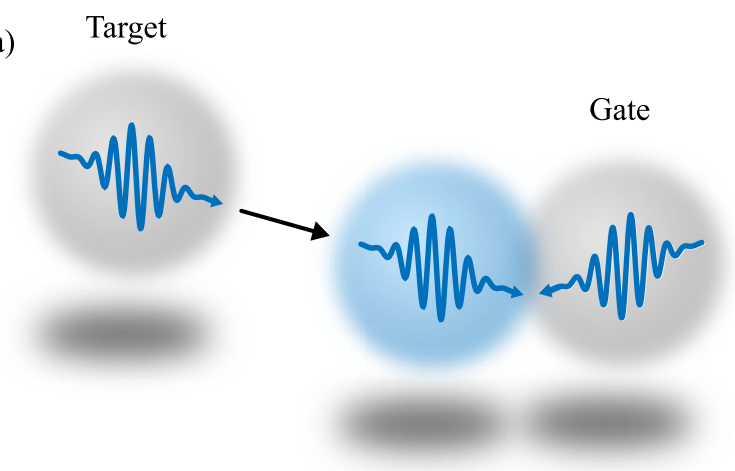

(b)

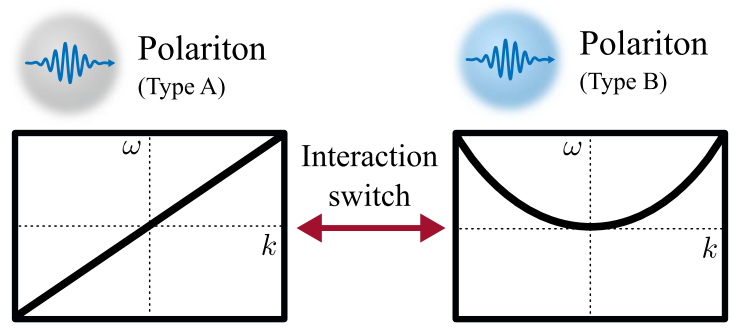

FIG. 1. Illustration of the basic principle of nonlinear polariton switching. (a) A photon (target) propagates initially as one type of dark-state polariton (type A, gray sphere), but is subsequently converted to another kind of dark-state polariton (type B, blue sphere) upon interacting with a second (gate) polariton. (b) This induces a change in the dispersion relation that governs the propagation of light and thereby mediates an effective photon interaction at greatly suppressed losses.

We outline a specific implementation that can be realized with minimal extensions to current experiments [49,55,56] and is shown to yield a conditional coupling between two distinct photonic modes. In particular, we show how this can be used to establish a reflective nonlinearity, in which a single photon stored in a Rydberg spin-wave excitation acts as an effective mirror, capable of reflecting photons with an arbitrary and continuously tunable reflection phase. The described realization of interaction-induced polariton switching can thus function as a single-photon router, facilitating a broad range of applications from quantum transistors to photonic gate operations. Finally, we discuss the performance of such applications based on current technology and in relation to previous blockade-based approaches.

\section{NONLINEAR POLARITON SWITCHING}

Rydberg dark-state polaritons acquire the properties of their constituents, inheriting kinetics from their photonic admixture and interactions from their atomic Rydberg-state component. Typically, these interactions are of a van der Waals type, causing a level shift $V(z)=C_{6} / z^{6}$ of the Rydberg state attached to one polariton when it interacts with another at a distance $z$. The most dramatic consequence of this effect is the Rydberg blockade [65], where the large induced level shift prevents the excitation of more than one Rydberg state within a so-called blockade radius. Since the van der Waals coefficient $C_{6} \sim n^{11}$ increases rapidly with the principal quantum number $n$ of the chosen Rydberg state [34], the available interaction strengths can vastly exceed any other energy scale in the system, and the available blockade radii can become significant. In current approaches to nonlinear optics based on Rydberg EIT, this blockade phenomenon is used to break EIT conditions and realize an effective polariton blockade [15,16,36,37,39-43].

On the contrary, we consider here a situation where this level shift is used rather to establish a switching mechanism between different types of dark-state polaritons. Consequently, the corresponding nonlinear optical response should thus be associated with minimal refraction and absorption, and only modify the dispersion relation that characterizes the photon propagation. As a specific example, we consider a situation in which the onset of interactions serves to cancel the linear dispersion of light and establish a locally quadratic dispersion, Fig. 1(b). This corresponds to a nonlinear switching between so-called slow-light $[46,47,66]$ and stationary-light [67-72] polaritons, both of which have been separately demonstrated in Refs. [73,74] and Refs. [75-77], respectively.

EIT is an effect that uses destructive interference between different excitation pathways to cancel the static optical response of a medium (which characterizes absorption and refraction). In the simplest realization of slow-light EIT, this typically involves a single control field to induce transparency for a second weak probe field on two-photon resonance with the transition to a stable excited atomic state. The transparency is associated with the formation of a dark-state polariton that is composed of the probe photons and the stable atomic excitations (spin waves) [46,47]. Because of its spin-wave component, the dark-state polaritons propagate at a greatly reduced speed according to the one-dimensional propagation equation [46,47],

$$
\partial_{t} \hat{\Psi}(z, t)=v \partial_{z} \hat{\Psi}(z, t),
$$

which describes the form-stable linear propagation of the slow-light polariton anihilation operator $\hat{\Psi}(z, t)$ with a group velocity $v$ that is typically much less than the vacuum speed of light $c$ [73].

Stationary light, on the other hand, can be realized when a pair of counterpropagating control fields is used to establish EIT for a pair of counterpropagating probe fields in a four-wave mixing configuration [67-72,75-77]. The resulting polaritons are then composed of both probe field modes, and become stationary when they contain an equal admixture of the two counterpropagating fields, such that their linear dispersions cancel each other to yield a leadingorder quadratic dispersion. This effectively endows these polaritons with a kinetic energy and mass [69,71], akin to 

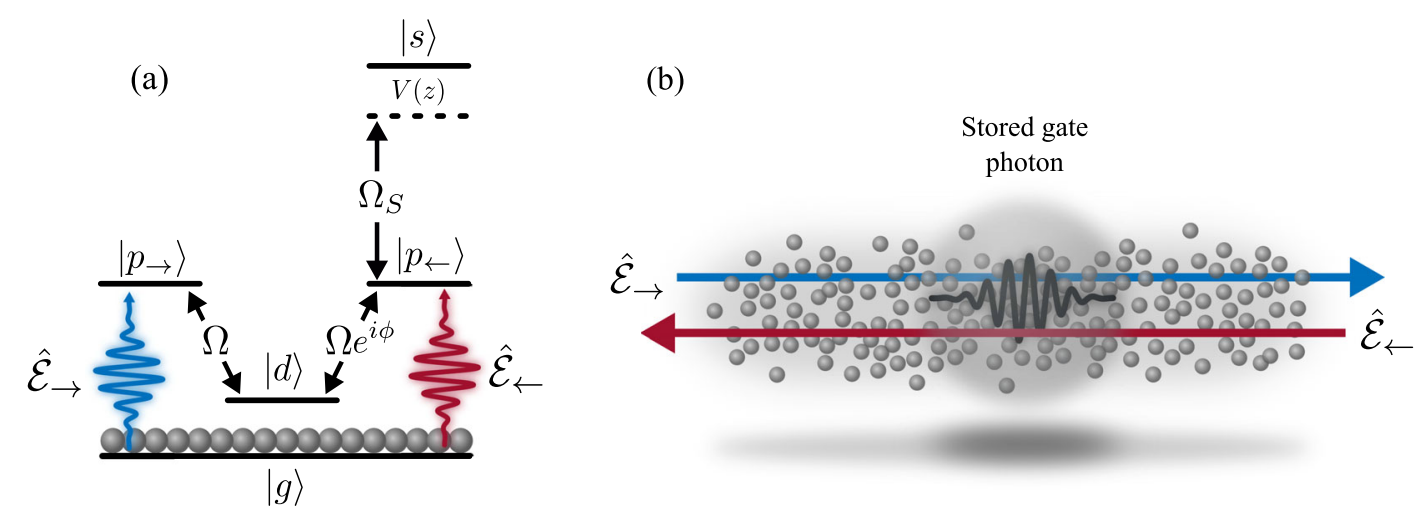

FIG. 2. (a) Schematics of the considered coupling scheme. Classical fields with indicated Rabi frequencies $\Omega$ and $\Omega_{S}$ establish EIT conditions for the counterpropagating photonic modes $\hat{\mathcal{E}}_{\rightarrow}$ and $\hat{\mathcal{E}}_{\leftarrow}$. The Rydberg state $|s\rangle$ is subject to a spatially dependent level shift $V(z)$ upon interacting with the stored gate excitation as illustrated in (b). This shift modifies the underlying EIT conditions for $\hat{\mathcal{E}}_{\rightarrow}$ and $\hat{\mathcal{E}}_{\leftarrow}$, rather than perturbing them.

massive particles. To leading order in the polariton bandwidth, the corresponding operator $\hat{\Phi}(z, t)$ describing the annihilation of a stationary-light polariton, thus, obeys the one-dimensional evolution equation [69,71],

$$
\partial_{t} \hat{\Phi}(z, t)=-\frac{1}{2 m} \partial_{z}^{2} \hat{\Phi}(z, t),
$$

where $m$ is the effective mass acquired by $\hat{\Phi}(z, t)$.

In order to engineer a Rydberg-mediated switching between the different types of polaritons described by Eqs. (1) and (2), we propose the level structure shown in Fig. 2(a). Here, EIT is achieved for two counterpropagating light fields described by the field operators $\hat{\mathcal{E}}_{\rightarrow}^{\dagger}$ and $\hat{\mathcal{E}}_{\leftarrow}^{\dagger}$, which create a probe photon in the right- and left-moving mode, respectively. As we see below, the precise nature of the resulting dark state is, however, controlled by the interaction-induced level shift of the Rydberg state $|s\rangle$ to which the fields are coupled. Specifically, our proposed coupling scheme is shown to support slow-light EIT conditions for $\hat{\mathcal{E}}_{\rightarrow}$ and $\hat{\mathcal{E}}_{\leftarrow}$ separately in the limit of weak interactions, while it facilitates stationary-light EIT under conditions of full Rydberg blockade.

\section{INTERACTION WITH A STORED SPIN WAVE}

The proposed polariton-switching mechanism is best analyzed by considering the conceptually simplest type of photon-photon interaction, whereby a (gate) photon is first stored in the atomic ensemble [47,78-80] as a collective Rydberg spin-wave excitation. Subsequently, a second (target) photon is sent through the medium and made to interact with the stored gate excitation [see Fig. 2(b)]. This approach provides a well-controlled way to engineer twophoton interactions [42], and has been demonstrated in a number of recent experiments [54-58].

The relevant atomic excitations are described by the continuous field operators [47] $\hat{P}_{\rightleftarrows}^{\dagger}(z, t), \hat{D}^{\dagger}(z, t)$, and $\hat{S}^{\dagger}(z, t)$, which create an excitation in $\left|p_{\rightleftarrows}\right\rangle,|d\rangle$, and $|s\rangle$, respectively, at position $z$. Moreover, we introduce the operator $\hat{S}_{g}^{\dagger}(z, t)$ that creates a stored gate excitation in an auxiliary Rydberg state $\left|s_{g}\right\rangle$ that is not laser coupled during the probe stage [42,54-58]. Along with $\hat{\mathcal{E}}_{\rightarrow}$ and $\hat{\mathcal{E}}_{\leftarrow}$, all of these field operators satisfy bosonic commutation relations [47].

In a rotating frame, the one-dimensional dynamics of this system are governed by the following (non-Hermitian) Hamiltonian:

$$
\begin{aligned}
\hat{H}= & -i c \int_{-\infty}^{\infty} d z\left[\hat{\mathcal{E}}_{\rightarrow}^{\dagger}(z) \partial_{z} \hat{\mathcal{E}}_{\rightarrow}(z)-\hat{\mathcal{E}}_{\leftarrow}^{\dagger}(z) \partial_{z} \hat{\mathcal{E}}_{\leftarrow}(z)\right]+G \int_{0}^{L} d z\left[\hat{\mathcal{E}}_{\rightarrow}^{\dagger}(z) \hat{P}_{\rightarrow}(z)+\hat{\mathcal{E}}_{\leftarrow}^{\dagger}(z) \hat{P}_{\leftarrow}(z)+\text { H.c. }\right] \\
& -i \gamma \int_{0}^{L} d z\left[\hat{P}_{\rightarrow}^{\dagger}(z) \hat{P}_{\rightarrow}(z)+\hat{P}_{\leftarrow}^{\dagger}(z) \hat{P}_{\leftarrow}(z)\right]+\Omega \int_{0}^{L} d z\left[\hat{D}^{\dagger}(z) \hat{P}_{\rightarrow}(z)+e^{i \phi} \hat{D}^{\dagger}(z) \hat{P}_{\leftarrow}(z)+\text { H.c. }\right] \\
& +\Omega_{S} \int_{0}^{L} d z\left[\hat{S}^{\dagger}(z) \hat{P}_{\leftarrow}(z)+\text { H.c. }\right]+\int_{0}^{L} \int_{0}^{L} d z d x V(z-x) \hat{S}_{g}^{\dagger}(x) \hat{S}^{\dagger}(z) \hat{S}(z) \hat{S}_{g}(x),
\end{aligned}
$$

where $L$ is the length of the medium. For simplicity, we assume that $\left|p_{\rightarrow}\right\rangle$ and $\left|p_{\leftarrow}\right\rangle$ decay with the scattering rate $2 \gamma$ and that the probe photon modes couple to their respective transitions with $G \equiv g \sqrt{\rho_{a}}$, where $\rho_{a}$ is the homogeneous atomic density 
and $g$ is the single atom coupling strength. The state $|d\rangle$ is coupled to $\left|p_{\rightarrow}\right\rangle$ and $\left|p_{\leftarrow}\right\rangle$ by classical control fields with identical Rabi frequencies $\Omega_{\rightarrow}=\Omega_{\leftarrow}=\Omega$, while we allow for a relative phase difference $\phi$ between them. Finally, the state $\left|p_{\leftarrow}\right\rangle$ is coupled by another classical field to the Rydberg state $|s\rangle$ with a Rabi frequency $\Omega_{S}$. The last term in Eq. (3) accounts for the spatially dependant level shift of the Rydberg state $|s\rangle$ due to its van der Waals interaction with the stored gate excitation. The typical range over which this shift affects the probe photon propagation can be characterized by the blockade radius $z_{b}$ according to $V\left(z_{b}\right)=\Omega_{S}^{2} / \gamma[42]$.

\section{POLARITON ANALYSIS}

Having established the basic idea and the specifics of the considered setup, let us now discuss the characteristics of the dark-state polaritons involved in the underlying switching protocol. The relevant dark-state polaritons can be identified as the zero-energy eigenstate solutions of the Hamiltonian Eq. (3) in the two limiting cases $V(z) \rightarrow 0$ and $V(z) \rightarrow \infty$, i.e., for vanishing interactions and in the limit of a complete Rydberg-state blockade.

Focusing first on the noninteracting situation, diagonalizing the system Hamiltonian in the absence of photon dispersion yields two dark-state polaritons of the form

$$
\begin{gathered}
\hat{\Psi}_{\rightarrow}=\frac{1}{\mathcal{N}_{\rightarrow}}\left[\Omega \Omega_{S} \hat{\mathcal{E}}_{\rightarrow}-G\left(\Omega_{S} \hat{D}-\Omega e^{i \phi} \hat{S}\right)\right], \\
\hat{\Psi}_{\leftarrow}=\frac{1}{\mathcal{N}_{\leftarrow}}\left[\Omega_{S} \hat{\mathcal{E}}_{\leftarrow}-G \hat{S}\right],
\end{gathered}
$$

where $\quad \mathcal{N}_{\rightarrow}=\sqrt{\Omega^{2} \Omega_{S}^{2}+G^{2}\left(\Omega^{2}+\Omega_{S}^{2}\right)} \quad$ and $\quad \mathcal{N}_{\leftarrow}=$ $\sqrt{G^{2}+\Omega_{S}^{2}}$ are the normalization factors required to obtain standard bosonic commutation relations for $\hat{\Psi}_{\rightarrow}$ and $\hat{\Psi}_{\leftarrow}$. These polaritons can be accredited to two separate slowlight EIT schemes supported simultaneously by the level structure in Fig. 2(a): the five-level system formed by $|g\rangle$, $\left|p_{\rightarrow}\right\rangle,|d\rangle,\left|p_{\leftarrow}\right\rangle$, and $|s\rangle$ (establishing EIT for $\hat{\mathcal{E}}_{\rightarrow}$ ), and the three-level system formed by $|g\rangle,\left|p_{\leftarrow}\right\rangle$, and $|s\rangle$ (establishing EIT for $\hat{\mathcal{E}}_{\leftarrow}$ ). We emphasize that the dark-state nature of $\hat{\Psi}_{\rightarrow}$ and $\hat{\Psi}_{\leftarrow}$ ensures that there is no coupling between the two underlying photonic modes. Hence, a right-moving input photon will undergo low-loss and form-stable propagation through the medium, and so will a left-moving photon.

The dispersion relations, $\omega_{\rightarrow}(k)$ and $\omega_{\leftarrow}(k)$, governing this propagation dynamics are readily obtained from a momentum space formulation of Eq. (3). To leading order in the photon momentum $k$ (and the ratio $\Omega / \Omega_{S}$ ), one finds

$$
\begin{gathered}
\omega_{\rightarrow}(k) \approx c\left[\frac{\Omega^{2}}{G^{2}+\Omega^{2}}\right] k+\mathcal{O}\left[k^{2}\right], \\
\omega_{\leftarrow}(k) \approx-c\left[\frac{\Omega_{S}^{2}}{G^{2}+\Omega_{S}^{2}}\right] k+\mathcal{O}\left[k^{2}\right] .
\end{gathered}
$$

As expected, one finds linear dispersion relations describing a form-stable propagation of the slow-light polaritons with group velocities $v_{\rightarrow}=\left(d \omega_{\rightarrow} / d k\right)$ and $v_{\leftarrow}=\left(d \omega_{\rightarrow} / d k\right)$, respectively. The two polaritons propagate in opposite directions with $v_{\rightarrow}=-\left(\Omega^{2} / \Omega_{S}^{2}\right) v_{\leftarrow}$ under the typical condition $G \gg \Omega, \Omega_{S}$. This is further illustrated in Fig. 3(a), where we plot the complete polariton spectrum admitted in this noninteracting situation, indeed revealing the emergence of two dark-state polariton branches at $k=0$ corresponding to $\hat{\Psi}_{\rightarrow}$ and $\hat{\Psi}_{\leftarrow}$.

Now we consider the polariton spectrum admitted well within a blockade radius away from the stored spin wave, i.e., under strong blockade conditions corresponding to $V(z) \rightarrow \infty$. In this case, the shifted Rydberg state exposes a modified effective level structure corresponding to a socalled dual- $V$ coupling scheme $[69,72]$, which can support stationary-light phenomena. For this system, one finds the emergence of only a single dark-state polariton $\hat{\Phi}$ of the type in Eq. (2). Diagonalizing the underlying system Hamiltonian Eq. (3), again in the absence of photon kinetics, one finds $\hat{\Phi}$ to be of the following form:

$$
\hat{\Phi}=\frac{1}{\mathcal{N}}\left[\Omega\left(\hat{\mathcal{E}}_{\rightarrow}+e^{i \phi} \hat{\mathcal{E}}_{\leftarrow}\right)-G \hat{D}\right],
$$

where $\mathcal{N}=\sqrt{\Omega^{2}+G^{2}}$ is the normalization factor. In contrast to the noninteracting limit, a coherent coupling is now established between the two optical modes $\hat{\mathcal{E}}_{\rightarrow}$ and $\hat{\mathcal{E}}_{\leftarrow}$. This is reflected in the photonic composition of $\hat{\Phi}$, which is composed of the symmetric superposition state of the optical fields, $\hat{\mathcal{E}}_{+}=(1 / \sqrt{2})\left(\hat{\mathcal{E}}_{\rightarrow}+e^{i \phi} \hat{\mathcal{E}}_{\leftarrow}\right)$.

The corresponding dispersion relation $\omega_{\leftrightarrow}(k)$ that governs the propagation of $\hat{\Phi}$ can be determined in a similar fashion as before, and reads

$$
\omega_{\leftrightarrow}(k) \approx-i 2 l_{\mathrm{abs}} \frac{c \Omega^{2}}{G^{2}+2 \Omega^{2}} k^{2}+\mathcal{O}\left[k^{3}\right]
$$

to lowest order in the photon momentum $k$, where $l_{\mathrm{abs}}=$ $c \gamma / G^{2}$ is the resonant two-level absorption length. Indeed, the obtained dispersion is quadratic in $k$, such that $\hat{\Phi}$ behaves as a stationary-light polariton. Figure 3(b) shows the complete polariton spectrum for $V(z) \rightarrow \infty$ and illustrates the above discussion of the dark-state polariton. 

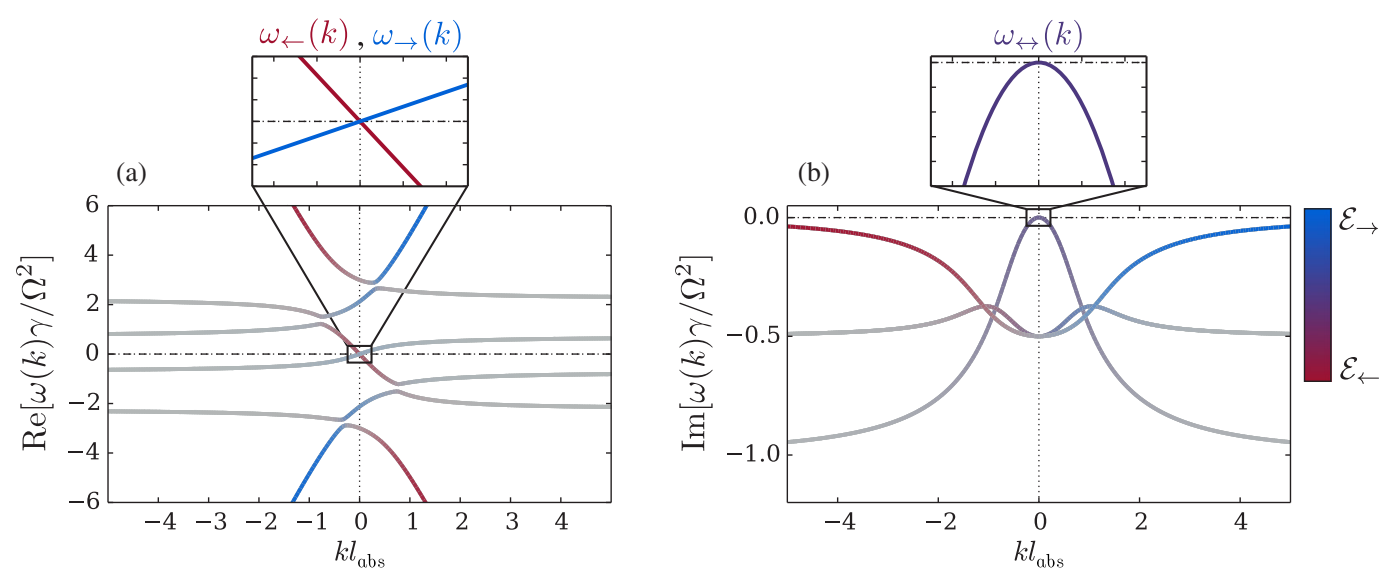

FIG. 3. (a) Real part of the polariton spectrum in the absence of interactions, indicating the emergence of two slow-light dark-state polaritons. These are separately governed by the linear dispersion relations $\omega_{\rightarrow}(k)$ and $\omega_{\leftarrow}(k)$ as given by Eqs. (6) and (7), respectively. Here, $\Omega_{S} / G=1, \Omega / G=0.5$, and $\gamma / \Omega=1$. (b) Imaginary part of the polariton spectrum for strong interactions, i.e., under conditions of a complete Rydberg blockade. In this case, one finds a single stationary-light dark-state polariton described by a quadratic dispersion relation $\omega_{\leftrightarrow}(k)$, Eq. (9). Here, $G / \Omega=1$ and $\gamma / \Omega=1$. For each bright-state branch there are two solutions with identical $\operatorname{Im}[\omega(k)]$. The blue to red color coding indicates the relative fraction of $\hat{\mathcal{E}}_{\rightarrow}$ and $\hat{\mathcal{E}}_{\leftarrow}$ comprising the underlying state of each polariton branch, while the gray scale indicates the overall atomic fraction.

\section{PHOTON PROPAGATION}

In order to develop an intuitive physical picture of the target photon dynamics, we first model the stored gate excitation as a spatially localized Rydberg impurity, and generalize this analysis to the consideration of a collective spin-wave state in Sec. VI. First, we transform into the Schrödinger picture. Introducing $|\psi(t)\rangle$ as the general timedependent wave function of the system, we define the twobody amplitudes $\mathcal{E}_{\rightarrow}(z, x, t)=\left\langle 0\left|\hat{\mathcal{E}}_{\rightarrow}^{\dagger}(z, t) \hat{S}_{g}^{\dagger}(x, t)\right| \psi\right\rangle$ and $\mathcal{E}_{\leftarrow}(z, x, t)=\left\langle 0\left|\hat{\mathcal{E}}_{\leftarrow}^{\dagger}(z, t) \hat{S}_{g}^{\dagger}(x, t)\right| \psi\right\rangle$ corresponding to a stored gate excitation at position $x$ and a target photon at position $z$ in the right- and left-moving mode, respectively. Denoting the temporal Fourier transform of $\mathcal{E}_{\rightarrow}(z, x, t)$ and $\mathcal{E}_{\leftarrow}(z, x, t)$ by $\tilde{\mathcal{E}}_{\rightarrow}(z, x, \omega)$ and $\tilde{\mathcal{E}}_{\leftarrow}(z, x, \omega)$, the photon dynamics can be formulated in terms of a matrix equation of the form

$$
i \partial_{z} \mathbf{E}(z, x, \omega)=\mathbf{M}(z-x, \omega) \mathbf{E}(z, x, \omega),
$$

where $\mathbf{E}(z, x, \omega)=\left\{\tilde{\mathcal{E}}_{\rightarrow}(z, x, \omega), \tilde{\mathcal{E}}_{\leftarrow}(z, x, \omega)\right\}$ and the propagation matrix is given by

$$
\mathbf{M}(z, \omega)=\left[\begin{array}{cc}
\chi_{\rightarrow}(z, \omega) & \chi(z, \omega) e^{i \phi} \\
-\chi(z, \omega) e^{-i \phi} & \chi_{\leftarrow}(z, \omega)
\end{array}\right] .
$$

The susceptibilities $\chi_{\rightarrow}(z, \omega)$ and $\chi_{\leftarrow}(z, \omega)$ characterize the propagation of $\tilde{\mathcal{E}}_{\rightarrow}$ and $\tilde{\mathcal{E}}_{\leftarrow}$, respectively, while $\chi(z, \omega)$ describes the coupling between the two modes. A derivation of Eq. (10) is outlined in Appendix A, along with the explicit expressions for the susceptibilities.

In the continuous wave $(\mathrm{cw})$ limit $(\omega \rightarrow 0)$ the propagation matrix in Eq. (11) can be parametrized in terms of a single susceptibility $\chi_{0}(z) \equiv \chi_{\rightarrow}(z, 0)=-\chi_{\leftarrow}(z, 0)=$ $-\chi(z, 0)$, given by

$$
\chi_{0}(z)=\frac{d_{b}}{\left(z / z_{b}\right)^{6}+2 i} .
$$

Here, we define $2 d_{b}=2 z_{b} / l_{\text {abs }}$ as the medium's optical depth per blockade radius. $\chi_{0}(z)$ basically characterizes an effective potential through which the stored spin wave can affect the target photon propagation dynamics. In particular, one finds that $\chi_{0}(z) \rightarrow 0$ outside the blockade radius of the stored excitation, $|z|>z_{b}$, consistent with the slowlight EIT conditions supported in this region and the associated decoupling of the photonic modes. However, $\chi_{0}(z)$ approaches $-i d_{b} / 2$ within the blockade volume, reflecting the fact that a coupling between these modes is established, which gives rise to stationary-light EIT conditions.

Considering a target photon incident on the medium from the left at $z=0$, its transmission and reflection can then be characterized by the following relations:

$$
\begin{aligned}
& \tilde{\mathcal{E}}_{\rightarrow}(L, x, \omega)=T_{n}(\omega, x) \tilde{\mathcal{E}}_{\rightarrow}(0, x, \omega), \\
& \tilde{\mathcal{E}}_{\leftarrow}(0, x, \omega)=R_{n}(\omega, x) \tilde{\mathcal{E}}_{\rightarrow}(0, x, \omega),
\end{aligned}
$$

where $T_{n}(\omega, x)$ and $R_{n}(\omega, x)$ are the transmission and reflection coefficients of the medium containing $n \in[0,1]$ stored gate excitations at position $x$.

Let us first consider the situation in which the gate excitation is absent. In this case, the target photon will initially generate the slow-light polariton described by $\hat{\Psi}_{\rightarrow}$ at the entrance of the medium. As we describe above, the 
formed polariton will then traverse the medium with a vanishing coupling to the counterpropagating mode and experience full transmission under perfect EIT conditions. The actual mechanism underlying this decoupling of $\hat{\mathcal{E}}_{\rightarrow}$ and $\hat{\mathcal{E}}_{\leftarrow}$ can be traced back to quantum interference effects involving the dressed states of the laser-driven Rydberg transition. Specifically, the resonant coupling of $\left|p_{\leftarrow}\right\rangle$ and $|s\rangle$ via the classical field $\Omega_{S}$ [see Fig. 2(a)] establishes a pair of light-shifted states,

$$
\left|f_{ \pm}\right\rangle=\frac{\left|p_{\leftarrow}\right\rangle \pm|s\rangle}{\sqrt{2}}
$$

which are shifted in energy by $\pm \Omega_{S}$, respectively. It is the destructive interference between competing excitation pathways involving these states that ultimately decouples the two modes of the target photon.

We note that this decoupling is exact on EIT resonance $(\omega=0)$ for any finite value of $\Omega_{S}$. However, this is not true for a finite bandwidth of the target photon. In this case, a nonvanishing coupling is established between the off-resonant frequency components of $\tilde{\mathcal{E}}_{\rightarrow}(z, x, \omega)$ and $\tilde{\mathcal{E}}_{\leftarrow}(z, x, \omega)$. Such bandwidth limitations exist for any realistic EIT setting, but can be minimized through a proper choice of parameters. To establish these conditions, we first expand $\left|T_{0}(\omega)\right|$ to lowest order in $\omega$ as $\left|T_{0}(\omega)\right| \approx 1-\left(\omega / \Delta \omega_{0}\right)^{2} . \Delta \omega_{0}$ then corresponds to the characteristic width of the transmission resonance, defining the range of frequencies over which the target photon is transparent, and is given explicitly as

$$
\Delta \omega_{0}=\Gamma\left[\left(1+2 \frac{\Omega^{2}}{\Omega_{S}^{2}}+2 \frac{\Omega^{4}}{\Omega_{S}^{4}}\right)+\frac{d}{2} \frac{\Omega^{4}}{\Omega_{S}^{4}}\right]^{-1 / 2},
$$

where $2 d=2 d_{b} L / z_{b}$ is the total optical depth of the medium and $\Gamma=\Omega^{2} / \gamma \sqrt{d}$.

According to Eq. (16), the transparency width tends to a maximum value of $\Delta \omega_{0} \rightarrow \Gamma$ when the conditions $2 \Omega^{2} / \Omega_{S}^{2} \ll 1$ and $d^{2} \Omega^{4} / 2 \Omega_{S}^{4} \ll 1$ are satisfied. The condition $d^{2} \Omega^{4} / 2 \Omega_{S}^{4} \ll 1$ accounts for a weak oscillatory behavior in $\left|T_{0}(\omega)\right|$. However, the effects of this only become observable at significant values of $d$, and can be safely neglected for our purposes. To understand the second condition, $2 \Omega^{2} / \Omega_{S}^{2} \ll 1$, first notice that in the limit $\Omega^{2} / \Omega_{S}^{2} \rightarrow \infty$ the Rydberg-state admixture of the $\hat{\Psi}_{\rightarrow}$ polariton vanishes, and the effective coupling scheme establishing EIT for $\hat{\mathcal{E}}_{\rightarrow}$ reduces to a $\Lambda$ system formed from $|g\rangle \leftrightarrow\left|p_{\rightarrow}\right\rangle \leftrightarrow|d\rangle$ [see Fig. 2(a)]. In this ideal limit, the optical response of the medium is then set solely by this effective EIT scheme with an effective transparency width of $\Omega^{2} / \gamma \sqrt{d}$ [47], which is consistent with the limiting value of $\Delta \omega_{0}$. Physically, though, $\Omega_{S}$ is required to be large since the effective decoupling of the photonic modes ultimately

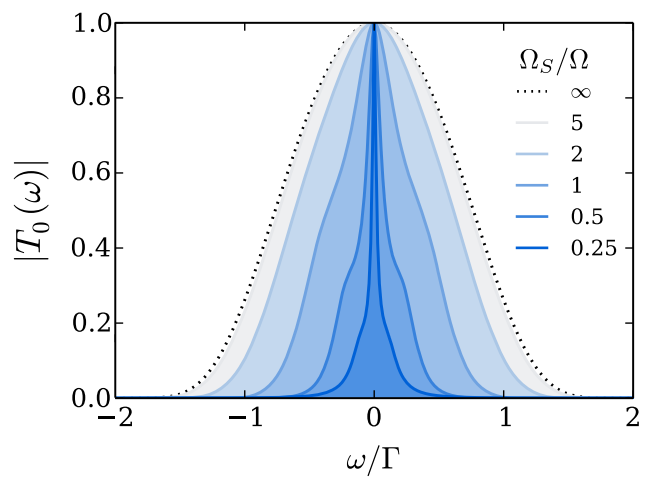

FIG. 4. Transmission coefficient in the absence of a stored gate excitation for various indicated values of $\Omega_{S} / \Omega$. The total optical depth is $2 d=50$, while $\gamma / \Omega=0.5$ and $G / \Omega=0.1$.

demands a large level splitting of the dressed states $\left|f_{ \pm}\right\rangle$, and this is given by $\pm \Omega_{S}$.

To verify this picture, we plot the solution for $T_{0}(\omega)$ in Fig. 4 for various ratios of $\Omega_{S} / \Omega$, and indeed find that the transmission spectrum converges to that of the $|g\rangle \leftrightarrow$ $\left|p_{\rightarrow}\right\rangle \leftrightarrow|d\rangle \Lambda$ system as $\Omega_{S}$ increases. Importantly, Fig. 4 demonstrates that near optimal transmission is already reached for remarkably small ratios of $\Omega_{S} / \Omega$, which are well within current experimental capabilities.

Let us now consider the propagation dynamics in the presence of a stored gate excitation. As before, upon entering the medium, the target photon propagates according to the linear dispersion relation $\omega_{\rightarrow}(k)$ in the form of a slow-light polariton $\hat{\Psi}_{\rightarrow}$. Upon entering the blockade volume established by the stored excitation, however, the target photon is subject to stationary-light EIT conditions, as described in the preceding sections. In this case, a coherent coupling between the $\hat{\mathcal{E}}_{\rightarrow}$ and $\hat{\mathcal{E}}_{\leftarrow}$ modes is established and the symmetric photonic state $\hat{\mathcal{E}}_{+}$is generated. Because of the lack of a linear dispersion, the photon can now only traverse the blockade region through a slow dispersion following $\omega_{\leftrightarrow}(k)=-i k^{2} / 2 m$. Upon crossing the blockade region the photon can reestablish the slowlight polariton $\hat{\Psi}_{\rightarrow}$ and be transmitted through the medium. However, there is typically a larger amplitude for the photon to diffuse into the counterpropagating direction and exit the medium as a $\hat{\Psi}_{\leftarrow}$ polariton, corresponding to reflection by the stored excitation. This reflection bias is essentially due to a boundary effect; the photon is more likely to diffuse the much shorter distance in the counterpropagating direction upon entering the interaction volume than diffuse the full $2 z_{b}$ length in the forward direction. The schematics of this overall process are depicted in Fig. 5(a).

To determine the relative importance of reflection and transmission, we can solve the propagation dynamics of the target photon exactly in the $\mathrm{cw}$ limit, for which the propagation matrix $\mathbf{M}$ in Eq. (11) is parametrized solely by $\chi_{0}(z)$, Eq. (12). With the boundary conditions 
(a)

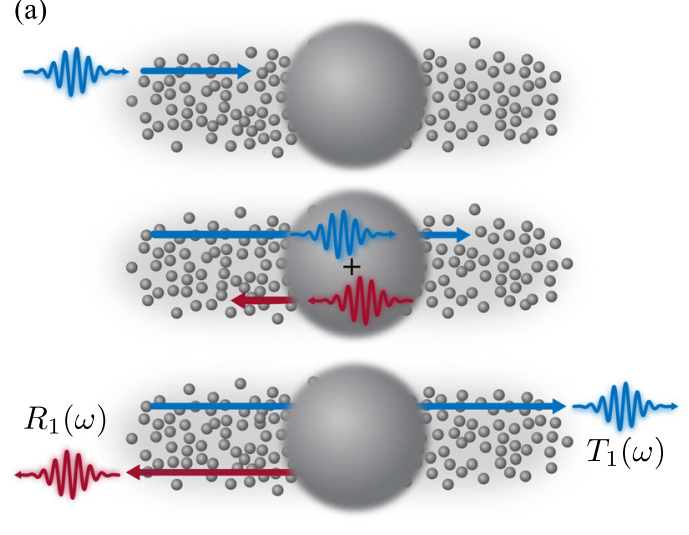

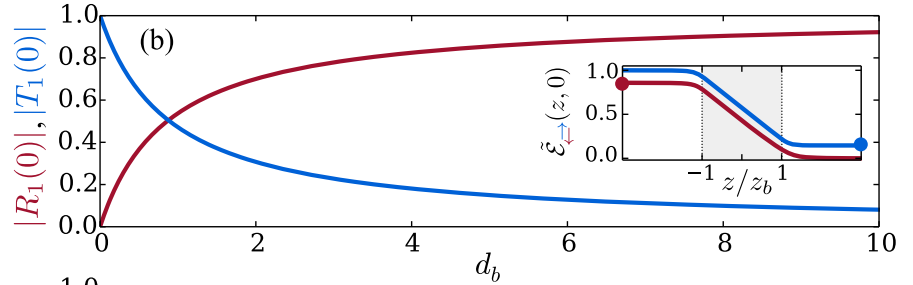

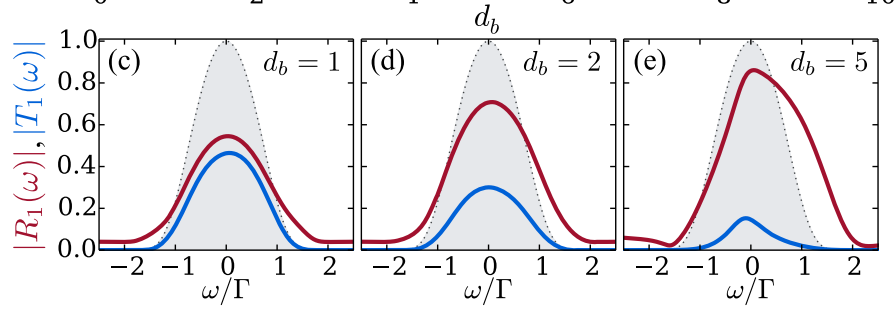

FIG. 5. (a) Illustration of target photon propagation in the presence of a stored gate excitation, where $R_{1}(\omega)$ and $T_{1}(\omega)$ are the reflection and transmission coefficients, respectively. (b) The resonant values of $R_{1}(\omega=0)$ and $T_{1}(\omega=0)$ are shown as a function of $d_{b}$ in red and blue, respectively. The inset shows the spatial dependence of the photonic amplitudes $\hat{\mathcal{E}}_{\rightarrow}(z)$ and $\hat{\mathcal{E}}_{\leftarrow}(z)$ within the medium for $d_{b}=5$, where the gray shaded region indicates the extent of the blockade volume established by the stored gate excitation. (c)-(e) The transmission and reflection spectra $T_{1}(\omega)$ and $R_{1}(\omega)$ are shown in blue and red, respectively, for various indicated values of $d_{b}$, where the stored excitation is located at the center of the medium. The total optical depth is $2 d=50$, and the remaining parameters are $\gamma / \Omega=0.5, \Omega / G=0.1, \Omega_{S} / G=0.5$. The transmission coefficient $T_{0}(\omega)$ in the absence of interactions is indicated by the gray shaded region for reference.

$\tilde{\mathcal{E}}_{\rightarrow}(z=0, x, 0)=\tilde{\mathcal{E}}_{0}$ and $\tilde{\mathcal{E}}_{\leftarrow}(z=L, x, 0)=0$, the solutions for the photonic fields can be readily obtained as

$$
\begin{aligned}
& \tilde{\mathcal{E}}_{\rightarrow}(z, x, 0)=\left[1-\frac{\nu(z, x)}{1+\nu(L, x)}\right] \tilde{\mathcal{E}}_{0}, \\
& \tilde{\mathcal{E}}_{\leftarrow}(z, x, 0)=e^{-i \phi}\left[\frac{\nu(L, x)-\nu(z, x)}{1+\nu(L, x)}\right] \tilde{\mathcal{E}}_{0},
\end{aligned}
$$

where we introduce $\nu(z, x)=i \int_{0}^{z} \chi_{0}\left(z^{\prime}-x\right) d z^{\prime}$. Assuming that the gate excitation is stored farther than $z_{b}$ away from the medium boundaries, the problem effectively becomes independent of the spin-wave position $x$. One can then write $\nu(L, x) \approx d_{b} \nu$, with $\nu=i \int_{-\infty}^{\infty} d z /\left(z^{6}+2 i\right)=$ $(\pi \sqrt[3]{1+i} / 3) \approx 1.1+0.3 i$, and obtain [from Eqs. (13) and (14)] the following simple expressions for the transmission and reflection coefficients:

$$
\begin{gathered}
T_{1}(\omega=0)=\frac{1}{1+\nu d_{b}}, \\
R_{1}(\omega=0)=\frac{\nu d_{b}}{1+\nu d_{b}} e^{-i \phi} .
\end{gathered}
$$

We plot $T_{1}(0)$ and $R_{1}(0)$ in Fig. 5(b), along with the spatial solutions of $\tilde{\mathcal{E}}_{\rightarrow}(z, 0)$ and $\tilde{\mathcal{E}}_{\leftarrow}(z, 0)$ within the medium.

The reflection amplitude increases with $d_{b}$, and eventually dominates the transmission beyond $d_{b} \approx 1$. Physically, this can be understood from the effective mass $m \propto 1 / l_{\text {abs }}$ [69] of the stationary-light polariton and the length $2 z_{b}$ of the blockade region. Since both increase with $d_{b}=z_{b} / l_{\mathrm{abs}}$, reflection dominates at large $d_{b}$, where traversing the entire blockade region is strongly suppressed such that photons predominantly exit in the opposite direction right at the incident boundary.

Additionally, Eq. (20) shows that one can imprint an arbitrary phase onto the reflected photon. This phase, $\phi$, is the relative phase difference between the classical control fields that establish stationary-light conditions within the interaction volume (see Fig. 2) and, therefore, can be well controlled and tuned in a continuous manner. For large $d_{b}$, the phase is a pure result of the reflection physics and does not depend on any system parameter other than the relative phase between the two classical control fields. Our setup can thus function as a photonic quantum router with an arbitrary and continuously tunable reflection phase. This robust conditional phase presents an important distinguishing feature compared to previous Rydberg-EIT-based protocols $[42,58]$.

Figure 5(c)-5(e) shows numerically obtained spectra $T_{1}(\omega)$ and $R_{1}(\omega)$ for various values of $d_{b}$. These are compared to the transmission spectrum $T_{0}(\omega)$ in the absence of the stored spin wave. As $d_{b}$ increases, one finds that the reflection spectrum develops an asymmetry. This asymmetry emerges since the Rydberg level shift $V(z)$ induces a nonsymmetric optical response, where the positive $(\omega>0)$ and negative $(\omega<0)$ frequency components are affected differently. However, the effects of this can be minimized by choosing a sufficiently narrow bandwidth of the target photon, as we discuss in Sec. VIII.

\section{COHERENCE PROPERTIES}

Another distinguishing feature of the described polaritonswitching mechanism is that it maintains EIT conditions and therefore operates at inherently suppressed photon losses. In 
Fig. 6(a), we show the loss $A=1-\left|T_{1}(0)\right|^{2}-\left|R_{1}(0)\right|^{2}$ as a function of $d_{b}$. Remarkably, absorption decreases with increasing $d_{b}$, even though the target photon is resonantly coupled to the medium. This, in turn, permits us to work under conditions of strong light-matter coupling, and stands in marked contrast to conventional Rydberg-EIT schemes where resonant photon coupling implies large interactioninduced losses, $A \approx 1-\exp \left[-4 d_{b}\right] \quad[42,54-57,62]$ [see Fig. 6(a)], and one requires large single-photon detunings for coherent nonlinear operations $[15,16,42,50]$.

Absorption in the current situation originates from the fact that the target photon does not switch fully adiabatically between the slow- and stationary-light polariton solutions. Specifically, this means that the target photon partially populates the bright-state polariton branches depicted in Fig. 3. However, the associated energy cost of $\sim G^{2} / \gamma=c l_{\text {abs }}^{-1}$ ensures that this population is suppressed with increasing $d_{b}$ and leads to the observed decrease of the loss coefficient $A$.

Thus far we have focused on the dynamics of the target photon, where it is sufficient to consider a localized gate excitation at a given position in the medium. Storage of the gate photon, however, generates a spatially delocalized collective spin-wave excitation, and the preservation of its coherent nature is essential for subsequent photon retrieval. Respectively, the retrieval efficiency is typically diminished by dissipative interactions with the incident target photon $[57,62,81]$, as we discuss below.

To this end, we consider the dynamics of the spinwave density matrix $\hat{\rho}(t):=\int_{0}^{L} d x \int_{0}^{L} d y \rho(x, y, t) \hat{S}_{g}^{\dagger}(x, t)|0\rangle$ $\langle 0| \hat{S}_{g}(y, t)$, where the complex elements $\rho(x, y, t)$ indicate the spatial coherence between spin-wave components at positions $x$ and $y$. Using the theoretical framework developed in Ref. [62], the final spin-wave state after the interaction with the target photon can be obtained exactly in the cw limit and is given by

$$
\begin{aligned}
\rho(x, y)= & \left\{1+i d_{b}\left[\frac{1}{1+\nu(L, x)}\right]\left[\frac{1}{1+\nu^{*}(L, y)}\right]\right. \\
& \left.\times \int_{0}^{L} d z \frac{(z-x)^{6}-(z-y)^{6}}{\left[(z-x)^{6}+2 i\right]\left[(z-y)^{6}-2 i\right]}\right\} \rho_{0}(x, y),
\end{aligned}
$$

where $\rho_{0}(x, y)$ denotes the initial (pure) state of the stored gate excitation. A detailed derivation of Eq. (21) is outlined in Appendix B.

While the spin-wave density $\rho(x, x)$ remains unaffected [62], target photon scattering results in partial decoherence, i.e., a reduction of the off-diagonal elements of $\rho(x, y)$. Assuming that the length of the medium is significantly

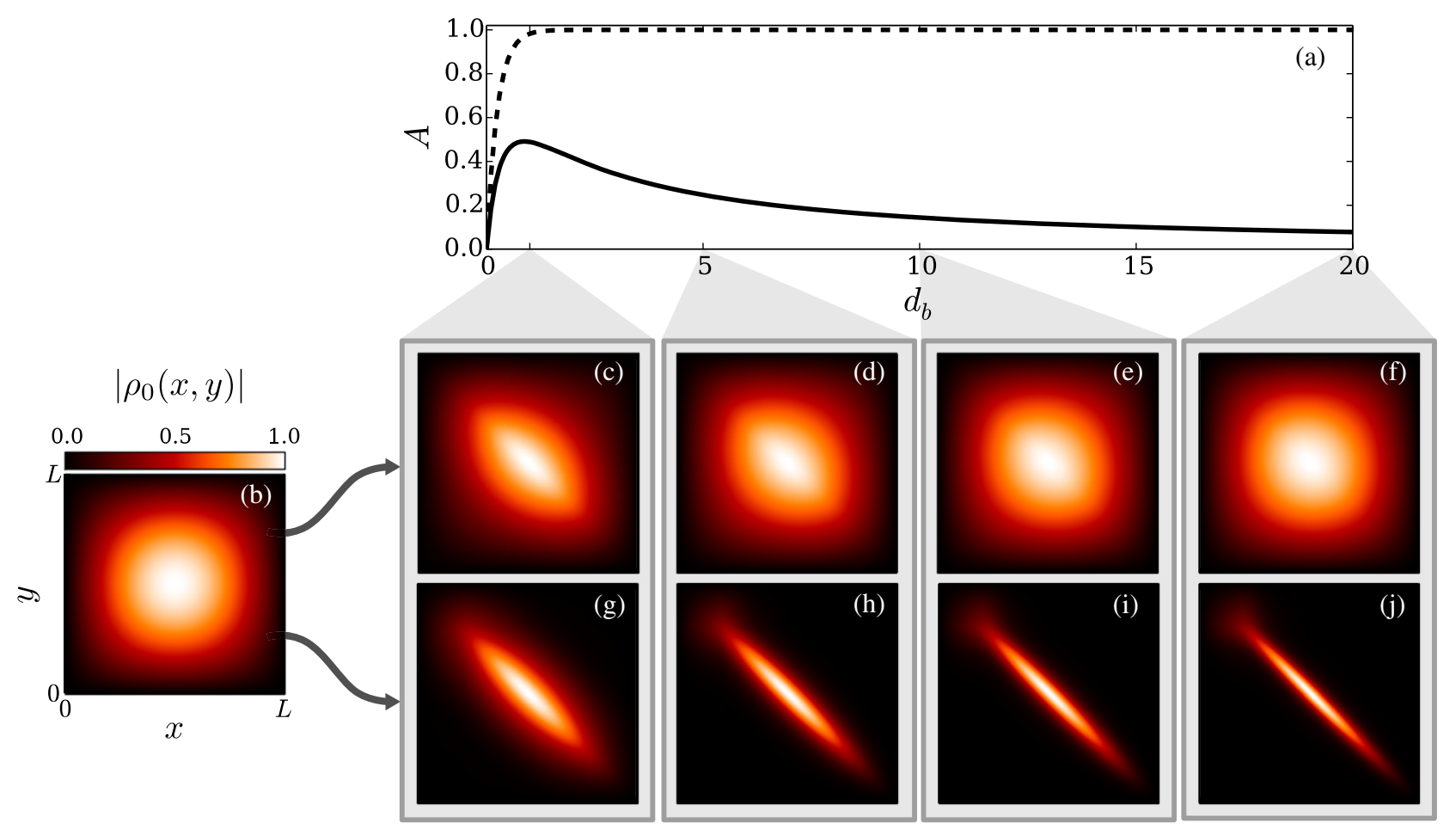

FIG. 6. (a) Loss coefficient $A$ as a function of $d_{b}$ (solid line) compared with the conventional polariton blockade mechanism (dashed line). (b) Initial density matrix, $\rho_{0}(x, y)=\sin (x \pi / L) \sin (y \pi / L)$, of the stored spin wave for $L=5 z_{b}$. (c)-(f) Final density matrix of the stored spin wave corresponding to the present coherent polariton-switching mechanism for various indicated values of $d_{b}$. Panels (g)-(j) show the final density matrix for the dissipative polariton blockade, which is shown to cause much stronger decoherence. 
longer than the extent of the spin-wave state, one can show that $\rho(x, y) \approx(1-A) \rho_{0}(x, y)$ at large distances $|x-y| \gg z_{b}$. Indeed, this shows explicitly that spin-wave decoherence is directly related to the nonlinear photon losses and, therefore, can be greatly suppressed by increasing $d_{b}$ in the present approach.

Figure 6 illustrates this difference between the present and conventional Rydberg-EIT schemes for an explicit initial spin wave with $\rho_{0}(x, y)=\sin (x \pi / L) \sin (y \pi / L)$ [see Fig. 6(b)]. In Figs. 6(c)-6(f), we show the final spin-wave state according to Eq. (21) for various values of $d_{b}$. As expected, the suppressed photon losses, shown by the solid line in Fig. 6(a), result in very little spin-wave decoherence, as reflected in a marginal deformation of $\rho(x, y)$, which reduces with increasing $d_{b}$. In Figs. $6(\mathrm{~g})-6(\mathrm{j})$, we plot the final spin-wave state corresponding to conventional Rydberg-EIT conditions [57,62,81]. In this case, the virtually complete scattering of the target field, as indicated by the dashed line in Fig. 6(a), causes strong decoherence that turns the initial spin wave into a near-classical distribution of the stored excitation.

\section{APPLICATIONS}

The demonstrated conditional reflection of the target field realizes a quantum nonlinear photon router $[11,82]$ in which a single gate photon can be used to control or redirect the flow of target photons between the two optical modes $\hat{\mathcal{E}}_{\rightarrow}$ and $\hat{\mathcal{E}}_{\leftarrow}$. This capability facilitates a broad range of functionalities.

The ability to modify the transmissive properties of the medium via gate storage has immediate practical applications in the context of optical switching [9,13,83-89]. Classical switching requires only a gatephoton-induced blocking of the target field transmission (via either dissipative scattering or coherent reflection), for which we can define a fidelity $\mathcal{F}_{\text {switch }}^{\text {(classical) }}=1-\left|T_{1}(0)\right|^{2}$, where $T_{1}(0)$ is given by Eq. (19). In previous Rydberg-EIT schemes $[42,54-57,62]$ relying on a dissipative polariton

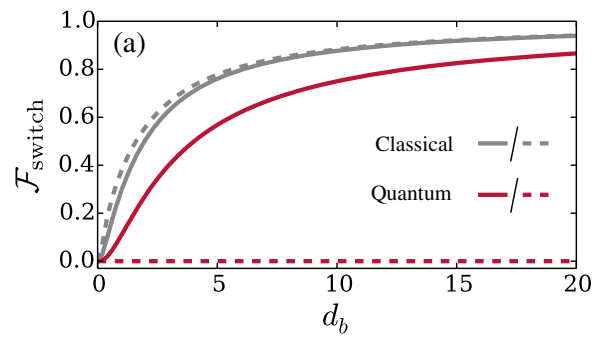

blockade, the switching fidelity is given by the nonlinear loss coefficient $\sim 1-\exp \left[-4 d_{b}\right]$ [42].

However, we emphasize that this purely dissipative nonlinearity fundamentally prevents the realization of a quantum switch, since the underlying photon scattering completely decoheres any quantum superposition state of the gate excitation. On the contrary, this does not affect the proposed routing approach, which does not rely on photon scattering. The quantum switching fidelity for the present scheme is given by the coherent reflection coefficient according to $\mathcal{F}_{\text {switch }}^{\text {(quantum) }}=\left|R_{1}(0)\right|^{2}$, with $R_{1}(0)$ given by Eq. (20).

The ability to retrieve the stored gate photon presents another essential performance aspect relevant for optical transistor operation or applications for nondestructive photon detection [54-57]. This factor is critically determined by dissipative scattering of target photons, as we discuss above. To account for the finite retrieval efficiency, we adopt the strategy of Ref. [62] and optimize the gate storage and retrieval efficiency $\eta$ in the presence of such decoherence processes by shaping the spatial profile of the stored spin-wave mode. We consider the target field in the $\mathrm{cw}$ limit, and describe the induced spin-wave decoherence by Eq. (21). Spin-wave decoherence for conventional Rydberg EIT is calculated according to Ref. [62]. The overall transistor fidelity is then given by the product of $\eta$ with the corresponding switching fidelity we discuss above.

In Fig. 7(a), we compare the different switch fidelities for the proposed photon router to the conventional approach based on nonlinear photon scattering. For classical operations, the fidelities are nearly identical since the apparent gain in the storage and retrieval efficiency due to reduced spin-wave decoherence is compensated by the higher switch fidelity in the dissipative case. More importantly, however, the proposed routing mechanism also functions as a quantum transistor, which otherwise is fundamentally impossible [62] for conventional Rydberg EIT. This is demonstrated by the red lines in Fig. 7(a).

As indicated by Eq. (20), the present approach permits us to imprint any relative phase between the applied control

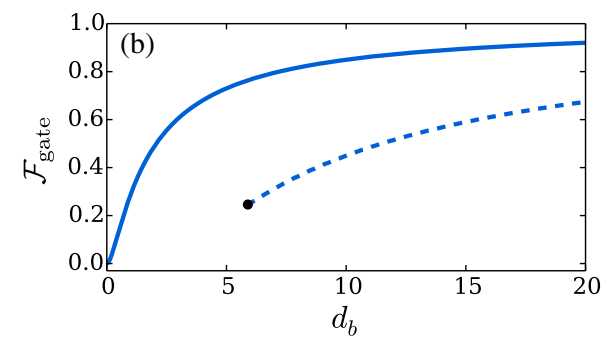

FIG. 7. (a) Overall switch fidelity, taking into account both the target field switching as well as the gate photon storage and retrieval efficiencies. The gray lines show the fidelity of a classical switch realized for a dissipative polariton blockade nonlinearity (dashed) and the coherent polariton switching nonlinearity (solid). The red lines show the corresponding fidelities for a quantum transistor. (b) Fidelity of a two-photon phase gate realized with the proposed photon router (solid line) compared with the corresponding fidelity of a $\pi$-phase gate based on the dispersive phase shift obtained from the polariton blockade mechanism (dashed line). The black dot indicates the critical value of $d_{b} \sim 6$ below which a dispersive phase shift of $\pi$ is fundamentally impossible with the polariton blockade mechanism under EIT conditions. 
fields onto the reflected target photon. In this way, the router can perform robust two-photon phase gate operations. The imprinted phase is largely independent of $d_{b}$, in contrast to previous schemes $[42,58]$ based on conventional Rydberg EIT. There, one requires a large single-photon detuning $\Delta$ to suppress the absorptive contribution to the nonlinear response, along with a large value of $d_{b}$ in order to account for the reduced single atom coupling and achieve a significant phase shift. In Fig. 7(b), we compare the resulting fidelity for a $\pi$-phase gate to the gate fidelity of the present scheme, $\mathcal{F}_{\text {gate }}=\left|R_{1}(0)\right|^{2}$ [see Eq. (20)]. Again, one finds a significant gain by the proposed routing mechanism for experimentally relevant parameters of moderate $d_{b}$. In Appendix $\mathrm{C}$, we present a more detailed discussion of the classical switch and phase gate based on the conventional polariton blockade mechanism.

\section{EXPERIMENTAL CONSIDERATIONS}

Finally, we discuss a concrete physical implementation of the described physics, using ${ }^{87} \mathrm{Rb}$ atoms as the most relevant example for current experiments [37,49,55,56]. Slow-light polaritons involving Rydberg states are being employed in a growing number of experiments $[15,16]$, and stationary-light polaritons as emerging from the double- $\Lambda$ coupling scheme of Fig. 2(a) have also been demonstrated experimentally $[76,90,91]$. Following this approach, the atomic cloud may be initialized in the $|g\rangle \equiv \mid 5 S_{1 / 2}, F=1$, $\left.m_{F}=0\right\rangle$ ground state. One can then choose the low-lying excited states $\left|p_{\rightarrow}\right\rangle$ and $\left|p_{\leftarrow}\right\rangle$ from the $\left|5 P_{3 / 2}, F=2\right\rangle$ hyperfine manifold. In this case, choosing the $\hat{\mathcal{E}}_{\rightarrow}$ and $\hat{\mathcal{E}}_{\leftarrow}$ fields to have $\sigma^{-}$and $\sigma^{+}$polarizations, respectively, $\hat{\mathcal{E}}_{\rightarrow}$ will drive the $|g\rangle \rightarrow\left|p_{\rightarrow}\right\rangle \equiv\left|5 P_{3 / 2}, F=2, m_{F}=-1\right\rangle$ transition, while $\hat{\mathcal{E}}_{\leftarrow}$ will drive the $|g\rangle \rightarrow\left|p_{\leftarrow}\right\rangle \equiv \mid 5 P_{3 / 2}$, $\left.F=2, m_{F}=+1\right\rangle$ transition. Stationary-light conditions are established by coupling these excited states back to the $|d\rangle \equiv\left|5 S_{1 / 2}, F=2, m_{F}=0\right\rangle$ hyperfine ground state by the counterpropagating classical fields with Rabi frequencies $\Omega_{\rightarrow}=\Omega$ and $\Omega_{\leftarrow}=\Omega e^{i \phi}$, as indicated in Fig. 2(a). We note that using $m_{F}=0$ ground states for $|g\rangle$ and $|d\rangle$ ensures that the classical Rabi frequencies have equal magnitude, along with the coupling strengths of $\hat{\mathcal{E}}_{\rightarrow}$ and $\hat{\mathcal{E}}_{\leftarrow}$. Finally, taking an $n S_{1 / 2}$ Rydberg state, $\sigma^{+}$-polarized light can be used to drive the $\left|p_{\leftarrow}\right\rangle \rightarrow|s\rangle=\left|n S_{1 / 2}, J=1 / 2, m_{J}=1 / 2\right\rangle$ transition.

Because of the degeneracy of the involved hyperfine and fine-structure manifolds, the optical fields would resonantly drive a number of additional transitions. These additional couplings can, however, be suppressed by applying a magnetic field along the light propagation axis. The resulting Zeeman shifts can be made sufficiently strong to isolate the desired transitions upon adjusting the field frequencies accordingly to maintain a resonant coupling. The resulting slight frequency shifts of $\hat{\mathcal{E}}_{\leftarrow}$ with respect to $\hat{\mathcal{E}}_{\rightarrow}$ can be straightforwardly compensated with standard techniques or by sending the reflected light through an identical medium without the Rydberg-state coupling. This permits us to reconvert $\hat{\mathcal{E}}_{\leftarrow}$ into $\hat{\mathcal{E}}_{\rightarrow}$ with an efficiency $\sim d^{2} /(1+d)^{2}$ that is much larger than that of the photon router.

In Fig. 8, we show the reflection efficiency,

$$
\mathcal{R}=\left.\left|\int_{-\infty}^{\infty} d \omega R_{1}(\omega)\right| \mathcal{E}_{0}(\omega)\right|^{2} \mid,
$$

for realistic experimental parameters of the described coupling scheme and different pulse lengths of the incoming target photon. Its spectrum $\mathcal{E}_{0}(\omega)$ is normalized according to $\int_{-\infty}^{\infty} d \omega\left|\mathcal{E}_{0}(\omega)\right|^{2}=1$. In calculating $\mathcal{R}$, we also account for a finite linewidth of the Rydberg state $\gamma_{S}$ by including an additional non-Hermitian term $-i \gamma_{S} \int d z \hat{S}^{\dagger}(z, t) \hat{S}(z, t)$ in the Hamiltonian of Eq. (3). We take a value of $\gamma_{S} / 2 \pi=300 \mathrm{kHz}$, which was used to describe recent experiments [92], accounting for both the natural linewidth of the Rydberg state and additional dephasing and decoherence processes. In spite of these additional effects, and the frequency asymmetry of $R_{1}(\omega)$ (see Fig. 5), the fidelity can approach the ideal limit $\left|R_{1}(\omega=0)\right|$ for realistic pulse lengths used in current experiments $[55,56,58]$.

Interestingly, a finite Rydberg decay rate does not directly lead to enhanced losses, as in the conventional setting, but instead causes enhanced reflection. This is because such decay no longer allows for a symmetric splitting of the dressed states $\left|f_{ \pm}\right\rangle$[cf. Eq. (15)] that is responsible for decoupling $\hat{\mathcal{E}}_{\rightarrow}$ and $\hat{\mathcal{E}}_{\leftarrow}$ in the limit of vanishing interactions. Stationary-light EIT is, however,

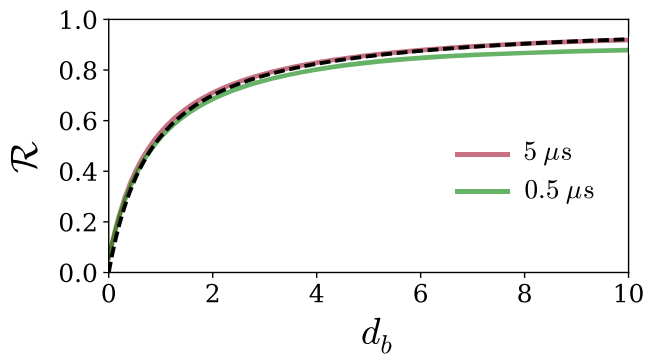

FIG. 8. Reflection efficiency, according to Eq. (22), for different indicated durations of a Gaussian incoming target pulse $\mathcal{E}_{0}$. The total optical depth is fixed at $2 d=50, \gamma / 2 \pi=3.05 \mathrm{MHz}$, and the classical Rabi frequencies are $\Omega / 2 \pi=5 \mathrm{MHz}$ and $\Omega_{S} / 2 \pi=20 \mathrm{MHz}$. The solid lines include a finite Rydberg-state linewidth $\gamma_{S} / 2 \pi=300 \mathrm{kHz}$ [92]. The gate excitation is located at the center of the medium and generates an interaction region with $z_{b}=\sqrt[6]{C_{6} \gamma / \Omega_{S}^{2}} \sim 8.7 \mu \mathrm{m}$, as obtained for the $100 S_{1 / 2}$ state of ${ }^{87} \mathrm{Rb}$ atoms [93]. The dashed line indicates the ideal limit of a vanishing photon bandwidth and $\gamma_{S}=0$, corresponding to Eq. (20). 
still supported for a finite $\gamma_{S}$, such that when the broadening of the Rydberg state becomes large, the entire medium thus behaves like a stationary-light medium and is associated with large reflection, rather than large absorption. However, for typical Rydberg decay rates, this unwanted reflection is negligible.

The Rydberg-state loss and decoherence also limits the target photon transmission in the absence of a stored gate excitation. For conventional Rydberg EIT, this is responsible for enhanced scattering, and the amplitude transmission decreases exponentially with the total optical depth $d$ according to $\left|T_{0}(0)\right| \approx \exp \left[-d\left(\gamma_{S} / \Gamma_{\mathrm{EIT}}\right)\right]$, where $\Gamma_{\mathrm{EIT}}=$ $\Omega_{S}^{2} / \gamma$ and $\gamma_{S} \ll \Gamma_{\text {EIT }}$ is assumed. However, for the present system, the transmission decreases polynomially as $\left|T_{0}(0)\right| \approx 1 /\left[1+d\left(\gamma_{S} / \Gamma_{\mathrm{EIT}}\right)\right]$, and therefore yields a substantial improvement for large $\gamma_{S}$. For the parameters of Fig. 8 this gives $\left|T_{0}(0)\right| \approx 0.95$, and thus does not limit the overall routing fidelity.

Finally, considering the finite spatial extent of both the target and the gate photon, one needs to account for another effect, namely, entanglement between the target field and the gate excitation emerging from the Rydberg-Rydberg atom interaction. More specifically, the reflection time of the target photon becomes correlated with the spatial position of the gate excitation in the atom cloud. While this presents another decoherence mechanism for the collective gate excitation, its actual effect is greatly suppressed as long as the target pulse length exceeds the characteristic difference of possible reflection times. The maximum time difference can be estimated as $\tau \approx$ $d\left(\gamma / \Omega^{2}+\gamma / \Omega_{S}^{2}\right)$ [47], which corresponds to the time delay between photons reflected at $z=0$ and $z=L$, respectively. Using the same parameters for $d, \gamma, \Omega$, and $\Omega_{S}$ as used in Fig. 8, we find that $\tau \sim 0.5 \mu$ s. This is an order of magnitude shorter than the largest pulse length in Fig. 8 and typical pulse lengths used in current experiments [55,58].

\section{SUMMARY AND CONCLUSIONS}

In summary, we devise a new approach to engineering effective photon interactions via particle interactions in an EIT medium. The basic principle is based on a modification rather than a breaking of EIT conditions to achieve a nonlinear alteration of light propagation under low-loss conditions. We present a specific implementation using laser-driven Rydberg-atom ensembles which realizes an effective photon-photon interaction that is reflective in character and highly coherent.

We demonstrate that in this way a single photon acts like a mirror with a robust and continuously tunable reflection phase and discuss a number of applications entailed by such a quantum nonlinear photon router. Here, the enhanced coherence properties of the developed polariton-switching mechanism offer a significant performance gain compared to existing approaches based on the
Rydberg blockade of dark-state polaritons [15,16,36,42]. This in turn permits us to achieve a strong optical response and high operational fidelities already at moderate values of $d_{b}$ that are well within the domain of accessible atomic densities, where additional decoherence processes [64] can be kept at a minimum.

The results of this work thus indicate that the exploration of nonlinearity mechanisms beyond the conventional Rydberg blockade holds new perspectives for all-optical quantum computing with Rydberg systems, such as the recent combination of EIT and dipolar Rydberg-state exchange interactions [92].

While we focus in this work on the interaction of a single propagating photon with a single stored gate excitation, the interaction of multiple freely propagating photons also holds interesting perspectives. The described type of nonlinearity could be used to coherently filter out highly nonclassical states of light from a classical light source, such as single-photon states $[43,49]$ or strongly correlated trains of photons [63]. As compared to the dissipative nonlinearity based on the polariton blockade considered in previous work $[43,49,63]$, the present coherent nonlinear reflection mechanism might, for example, require significantly lower optical densities for generating spatially ordered photons [63] and, thereby, make such exotic states of light accessible with present experimental capabilities.

Moreover, the broken left-right symmetry of the underlying coupling scheme in Fig. 2(a) implies that the optical response of the medium acquires a strong dependence on the propagation direction of the light. This effect can be investigated in multiphoton transmission measurements, where, for example, a head-on collision of two photons would generate a pair of copropagating photons with a strong bias in one direction. We emphasize that this symmetry breaking is a nonlinear effect, which is in contrast to the chiral linear response currently being explored in nanoscale waveguides [94-97]. The availability of such unusual types of photon interactions combined with the freely tunable reflection phase suggests intriguing perspectives for the collective engineering of nonclassical multiphoton states or the exploration of exotic many-body physics with photons.

\section{ACKNOWLEDGMENTS}

We thank C. S. Adams, M. Baghery, H. Gorniaczyk, M. Gullans, S. Hofferberth, D. Paredes-Barato, and E. Zeuthen for valuable discussions. This work was funded by the EU through the FET Grant No. 512862 (HAIRS), and the H2020-FETPROACT-2014 Grant No. 640378 (RySQ), by the DFG through the SPP 1929, and by the DNRF.

\section{APPENDIX A: PHOTON PROPAGATION EQUATIONS}

Here, we outline the derivation of the propagation equations in Eq. (10) and provide explicit expressions 
for the susceptibility functions appearing in Eq. (11). Starting from the Heisenberg equations derived from the Hamiltonian in Eq. (3), one obtains equations of motion for all relevant two-body amplitudes describing a stored gate excitation at position $x$ and a target excitation at position $z$. Using the shorthand notation $\mathcal{E}_{\rightarrow} \equiv \mathcal{E}_{\rightarrow}(z, x, t)$, $\mathcal{E}_{\leftarrow} \equiv \mathcal{E}_{\leftarrow}(z, x, t)$, etc. for convenience, these coupled equations of motion can be written as

$$
\begin{gathered}
\partial_{t} \mathcal{E}_{\rightarrow}=-c \partial_{z} \mathcal{E}_{\rightarrow}-i G P_{\rightarrow}, \\
\partial_{t} \mathcal{E}_{\leftarrow}=c \partial_{z} \mathcal{E}_{\leftarrow}-i G P_{\leftarrow}, \\
\partial_{t} P_{\rightarrow}=-i G \mathcal{E}_{\rightarrow}-i \Omega D-\gamma P_{\rightarrow}, \\
\partial_{t} P_{\leftarrow}=-i G \mathcal{E}_{\leftarrow}-i \Omega e^{-i \phi} D-i \Omega_{S} S-\gamma P_{\leftarrow}, \\
\partial_{t} D=-i \Omega\left(P_{\rightarrow}+e^{i \phi} P_{\leftarrow}\right), \\
\partial_{t} S=-i \Omega_{S} P_{\leftarrow}-i V(z-x) S .
\end{gathered}
$$

Transforming to frequency space, we then obtain a series of equations for $\tilde{\mathcal{E}}_{\rightarrow}(z, x, \omega)=(2 \pi)^{-1 / 2} \int_{-\infty}^{\infty} d t e^{-i \omega t} \mathcal{E}_{\rightarrow}(z, x, t)$, $\tilde{\mathcal{E}}_{\leftarrow}(z, x, \omega)=(2 \pi)^{-1 / 2} \int_{-\infty}^{\infty} d t e^{-i \omega t} \mathcal{E}_{\leftarrow}(z, x, t)$ etc. Upon solving for $\tilde{P}_{\rightarrow}(z, x, \omega)$ and $\tilde{P}_{\leftarrow}(z, x, \omega)$ in terms of the photonic amplitudes, and inserting these into Eqs. (A1) and (A2), one immediately arrives at a closed system of equations for $\tilde{\mathcal{E}}_{\rightarrow}(z, x, \omega)$ and $\tilde{\mathcal{E}}_{\leftarrow}(z, x, \omega)$ as given by Eqs. (10) and (11). The explicit expressions for $\chi_{\rightarrow}(z, \omega), \chi_{\leftarrow}(z, \omega)$, and $\chi(z, \omega)$ appearing in Eq. (11) are given by

$$
\begin{gathered}
\chi_{\rightarrow}(z, \omega)=-\frac{\omega}{c}+\frac{G^{2}}{c} \frac{\left(\xi-\frac{\Omega_{S}^{2}}{\omega-V(z)}\right)}{\xi\left(\xi-\frac{\Omega_{S}^{2}}{\omega-V(z)}\right)-\frac{\Omega^{4}}{\omega^{2}}}, \\
\chi_{\leftarrow}(z, \omega)=\frac{\omega}{c}-\frac{G^{2}}{c} \frac{\xi}{\xi\left(\xi-\frac{\Omega_{S}^{2}}{\omega-V(z)}\right)-\frac{\Omega^{4}}{\omega^{2}}}, \\
\chi(z, \omega)=\frac{G^{2}}{c} \frac{\Omega^{2}}{\omega} \frac{1}{\xi\left(\xi-\frac{\Omega_{S}^{2}}{\omega-V(z)}\right)-\frac{\Omega^{4}}{\omega^{2}}},
\end{gathered}
$$

where we introduce $\xi=\omega+i \gamma-\Omega^{2} / \omega$.

\section{APPENDIX B: SPIN-WAVE DECOHERENCE DYNAMICS}

Here, we outline the solution to the scattering-induced decoherence dynamics of the stored gate spin wave, as given by Eq. (21). To this end, we begin with the equation of motion for the density matrix elements, $\rho(x, y, t)$, of the stored spin wave, which can be expressed as

$$
\begin{aligned}
\partial_{t} \rho(x, y, t)= & i \int_{0}^{L} d z[V(z-x)-V(z-y)] \\
& \times S^{*}(z, x, t) S(z, y, t) .
\end{aligned}
$$

$S(z, x, t)$ is again the two-body probability amplitude to have an $|s\rangle$ Rydberg excitation at position $z$ and a stored gate excitation at position $x$. Imposing the initial condition $\rho(x, y, 0)=\rho_{0}(x, y)$, the general solution to Eq. (B1) is given by

$$
\begin{aligned}
\rho(x, y, t)= & \left\{1+i \int_{0}^{L} d z[V(z-x)-V(z-y)]\right. \\
& \left.\times \int_{-\infty}^{t} d \tau S^{*}(z, x, \tau) S(z, y, \tau)\right\} \rho_{0}(x, y) .
\end{aligned}
$$

Solving the target field dynamics governed by Eqs. (A1)-(A6) in the cw limit, one can obtain a solution for the Rydberg spin-wave amplitude $S(z, x)$ in terms of the photonic amplitudes:

$$
S(z, x)=\frac{G \Omega_{S}}{\gamma} \frac{\mathcal{E}_{\leftarrow}(z, x)-\mathcal{E}_{\rightarrow}(z, x)}{\Omega_{S}^{2} / \gamma+2 i V(z-x)} .
$$

Using the solutions for $\mathcal{E}_{\rightarrow}(z, x)$ and $\mathcal{E}_{\leftarrow}(z, x)$ according to Eqs. (17) and (18), the target spin-wave amplitude can be written as

$S(z, x)=-\frac{G \Omega_{S}}{\gamma} \frac{1}{\Omega_{S}^{2} / \gamma+2 i V(z-x)} \frac{1}{1+\nu(L, x)} \mathcal{E}_{0}$,

where the amplitude $\mathcal{E}_{0}$ describes the incoming target photon, with the normalization $\int_{-\infty}^{\infty} d t\left|\mathcal{E}_{0}\right|^{2}=1 / c$. Inserting this result into Eq. (B2) and carrying out the time integration, one arrives at the result given in Eq. (20), where distances have been rescaled by $z_{b}$, defined according to $V\left(z_{b}\right)=\Omega_{S}^{2} / \gamma$.

Let us finally consider Eq. (21) in the limit $|x-y| \gg z_{b}$. In this case, the spatial integral has two separate contributions around $z \sim x$ and $z \sim y$, such that the solution for $\rho(x, y)$ can be approximated by

$\rho(x, y) \approx\left\{1-\frac{\nu(L, x)+\nu^{*}(L, y)}{[1+\nu(L, x)]\left[1+\nu^{*}(L, y)\right]}\right\} \rho_{0}(x, y)$.

Assuming further that the medium length $L$ greatly exceeds the spatial extent of the gate spin wave, we can use $\nu(L, x)=\nu(L, y)=d_{b} \nu$ and obtain the expression

$$
\begin{aligned}
\rho(x, y) & \approx\left\{1-\frac{2 d_{b} \operatorname{Re}[\nu]}{\left|1+d_{b} \nu\right|^{2}}\right\} \rho_{0}(x, y) \\
& =(1-A) \rho_{0}(x, y),
\end{aligned}
$$


discussed in the main text, where $A$ is the loss coefficient introduced in Sec. VI.

\section{APPENDIX C: CONVENTIONAL POLARITON BLOCKADE}

Here, we present a description of the polariton blockade mechanism for mediating a target gate interaction, which is used to draw the comparisons shown in Fig. 7. In this situation, following the storage of a gate photon, a target photon is made to propagate under standard Rydberg-EIT conditions based on a three-level ladder scheme with a single-photon detuning $\Delta$ [36]. In the $\mathrm{cw}$ limit, the amplitude $\mathcal{E}(z)$ of the target photon follows a propagation equation of the form $[16,42]$

$$
\partial_{z} \mathcal{E}(z)=-\chi_{\mathrm{bl}}(\Delta, z) \mathcal{E}(z)
$$

where the susceptibility $\chi_{\mathrm{bl}}(\Delta, z)$ is given by

$$
\chi_{\mathrm{bl}}(\Delta, z)=\frac{1}{l_{\mathrm{abs}}} \frac{\gamma}{\Delta} \frac{V(z)}{\left[\frac{\gamma}{\Delta}-i\right] V(z)-i \frac{\Omega^{2}}{\Delta}},
$$

for a stored gate excitation at $z=0$. Assuming that the medium length $L$ is much longer than the blockade radius, the phase shift $\phi(\Delta)$ and amplitude attenuation $\exp [-\kappa(\Delta)]$ of the transmitted target photon can then be calculated as [42]

$$
\begin{aligned}
& \phi(\Delta)=-\operatorname{Im}\left[\int_{-\infty}^{\infty} d z \chi_{\mathrm{bl}}(\Delta, z)\right], \\
& \kappa(\Delta)=\operatorname{Re}\left[\int_{-\infty}^{\infty} d z \chi_{\mathrm{bl}}(\Delta, z)\right] .
\end{aligned}
$$

On single-photon resonance $\Delta=0$, the associated loss $1-\exp [-2 \kappa(0)]$ corresponds to the classical switching fidelity depicted by the gray dashed line shown in Fig. 7(a). Conversely, to determine the phase gate fidelity we set $\phi(\Delta)=\pi$ and substitute the corresponding value for $\Delta$ into Eq. (C4). This yields the fidelity $1-\exp [-2 \kappa(\Delta)]$, which is shown by the dashed line in Fig. 7(b). We note that for $d_{b} \lesssim 6$, the optical depth is not sufficient to achieve a $\pi$-phase shift. i.e., there is no real solution to $\phi(\Delta)=\pi$, which explains the vanishing fidelity over this region in Fig. 7(b).

To leading order in $\gamma / \Delta$, one obtains the analytical expressions

$$
\begin{gathered}
\phi(\Delta)=-d_{b} \frac{\gamma}{\Delta} \frac{2 \pi}{3}, \\
\kappa(\Delta)=d_{b}\left(\frac{\gamma}{\Delta}\right)^{2} \frac{5 \pi}{9} .
\end{gathered}
$$

For large $d_{b}$, the gate infidelity is thus given by $1-\mathcal{F}_{\text {gate }} \approx 5 \pi /\left(2 d_{b}\right)$. This is the same scaling as for $1-\left|R_{1}\right|^{2} \approx 1.69 / d_{b}$ obtained from Eq. (20), which, however, features a significantly smaller prefactor.

[1] D. E. Chang, V. Vuletić, and M. D. Lukin, Quantum Nonlinear Optics-Photon by Photon, Nat. Photonics 8, 685 (2014).

[2] A. Reiserer and G. Rempe, Cavity-Based Quantum Networks with Single Atoms and Optical Photons, Rev. Mod. Phys. 87, 1379 (2015).

[3] A. Reiserer and G. Rempe, Nondestructive Detection of an Optical Photon, Science 342, 1349 (2013).

[4] A. Reiserer, N. Kalb, G. Rempe, and S. Ritter, A Quantum Gate between a Flying Optical Photon and a Single Trapped Atom, Nature (London) 508, 237 (2014).

[5] E. Shahmoon and G. Kurizki, Nonlinear Theory of LaserInduced Dipolar Interactions in Arbitrary Geometry, Phys. Rev. A 89, 043419 (2014).

[6] P. Lodahl, S. Mahmoodian, and S. Stobbe, Interfacing Single Photons and Single Quantum Dots with Photonic Nanostructures, Rev. Mod. Phys. 87, 347 (2015).

[7] A. Javadi, I. Söllner, M. Arcari, S. Lindskov Hansen, L. Midolo, S. Mahmoodian, G Kiršansk, T. Pregnolato, E. H. Lee, J. D. Song, S. Stobbe, and P. Lodahl, Single-Photon Non-Linear Optics with a Quantum Dot in a Waveguide, Nat. Commun. 6, 8655 (2015).

[8] I. Söllner, S. Mahmoodian, S. L. Hansen, L. Midolo, A. Javadi, G. Kiršansk, T. Pregnolato, H. El-Ella, E. H. Lee, J. D. Song, S. Stobbe, and P. Lodahl, Deterministic PhotonEmitter Coupling in Chiral Photonic Circuits, Nat. Nanotechnol. 10, 775 (2015).

[9] J. Hwang, M. Pototschnig, R. Lettow, G. Zumofen, A. Renn, S. Götzinger, and V. Sandoghdar, A Single-Molecule Optical Transistor, Nature (London) 460, 76 (2009).

[10] A. Maser, B. Gmeiner, T. Utikal, S. Götzinger, and V. Sandoghdar, Few-Photon Coherent Nonlinear Optics with a Single Molecule, Nat. Photonics 10, 450 (2016).

[11] I. Shomroni, S. Rosenblum, Y. Lovsky, O. Bechler, G. Guendelman, and B. Dayan, Quantum Optics. All-Optical Routing of Single Photons by a One-Atom Switch Controlled by a Single Photon, Science 345, 903 (2014).

[12] J. D. Thompson, T. G. Tiecke, N. P. de Leon, J. Feist, A. V. Akimov, M. Gullans, A. S. Zibrov, V. Vuletić, and M. D. Lukin, Coupling a Single Trapped Atom to a Nanoscale Optical Cavity, Science 340, 1202 (2013).

[13] T. G. Tiecke, J. D. Thompson, N. P. de Leon, L. R. Liu, V. Vuletić, and M.D. Lukin, Nanophotonic Quantum Phase Switch with a Single Atom, Nature (London) 508, 241 (2014).

[14] A. Goban, C.-L. Hung, S.-P. Yu, J. D. Hood, J. A. Muniz, J. H. Lee, M. J. Martin, A. C. McClung, K. S. Choi, D. E. Chang, O. Painter, and H. J. Kimble, Atom-Light Interactions in Photonic Crystals, Nat. Commun. 5, 3808 (2014).

[15] O. Firstenberg, C. S. Adams, and S. Hofferberth, Nonlinear Quantum Optics Mediated by Rydberg Interactions, J. Phys. B 49, 152003 (2016). 
[16] C. Murray and T. Pohl, Advances In Atomic, Molecular, and Optical Physics (Academic Press, New York, 2016), Vol. 65, Chap. 7, pp. 321-372.

[17] J. S. Douglas, H. Habibian, C.-L. Hung, A. V. Gorshkov, H. J. Kimble, and D. E. Chang, Quantum Many-Body Models with Cold Atoms Coupled to Photonic Crystals, Nat. Photonics 9, 326 (2015).

[18] Y. Shi, Z. Yu, and S. Fan, Limitations of Nonlinear Optical Isolators due to Dynamic Reciprocity, Nat. Photonics 9, 388 (2015).

[19] E. Shahmoon, P. Grišins, H. P. Stimming, I. Mazets, and G. Kurizki, Highly Nonlocal Optical Nonlinearities in Atoms Trapped Near a Waveguide, Optica 3, 725 (2016).

[20] J. S. Douglas, T. Caneva, and D. E. Chang, Photon Molecules in Atomic Gases Trapped Near Photonic Crystal Waveguides, Phys. Rev. X 6, 031017 (2016).

[21] K. Singer, M. Reetz-Lamour, T. Amthor, L. G. Marcassa, and M. Weidemüller, Suppression of Excitation and Spectral Broadening Induced by Interactions in a Cold Gas of Rydberg Atoms, Phys. Rev. Lett. 93, 163001 (2004).

[22] D. Tong, S. M. Farooqi, J. Stanojevic, S. Krishnan, Y. P. Zhang, R. Côté, E. E. Eyler, and P. L. Gould, Local Blockade of Rydberg Excitation in an Ultracold Gas, Phys. Rev. Lett. 93, 063001 (2004).

[23] T. Cubel Liebisch, A. Reinhard, P. R. Berman, and G. Raithel, Atom Counting Statistics in Ensembles of Interacting Rydberg Atoms, Phys. Rev. Lett. 95, 253002 (2005).

[24] R. Heidemann, U. Raitzsch, V. Bendkowsky, B. Butscher, R. Löw, L. Santos, and T. Pfau, Evidence for Coherent Collective Rydberg Excitation in the Strong Blockade Regime, Phys. Rev. Lett. 99, 163601 (2007).

[25] L. Isenhower, E. Urban, X. L. Zhang, A. T. Gill, T. Henage, T. A. Johnson, T. G. Walker, and M. Saffman, Demonstration of a Neutral Atom Controlled-NOT Quantum Gate, Phys. Rev. Lett. 104, 010503 (2010).

[26] T. Wilk, A. Gaëtan, C. Evellin, J. Wolters, Y. Miroshnychenko, P. Grangier, and A. Browaeys, Entanglement of Two Individual Neutral Atoms Using Rydberg Blockade, Phys. Rev. Lett. 104, 010502 (2010).

[27] M. Viteau, M. G. Bason, J. Radogostowicz, N. Malossi, D. Ciampini, O. Morsch, and E. Arimondo, Rydberg Excitations in Bose-Einstein Condensates in Quasi-OneDimensional Potentials and Optical Lattices, Phys. Rev. Lett. 107, 060402 (2011).

[28] M. Ebert, A. Gill, M. Gibbons, X. Zhang, M. Saffman, and T. G. Walker, Atomic Fock State Preparation Using Rydberg Blockade, Phys. Rev. Lett. 112, 043602 (2014).

[29] H. Schempp, G. Günter, M. Robert-de-Saint-Vincent, C. S. Hofmann, D. Breyel, A. Komnik, D. W. Schönleber, M. Gärttner, J. Evers, S. Whitlock, and M. Weidemüller, Full Counting Statistics of Laser Excited Rydberg Aggregates in a One-Dimensional Geometry, Phys. Rev. Lett. 112, 013002 (2014).

[30] N. Malossi, M. M. Valado, S. Scotto, P. Huillery, P. Pillet, D. Ciampini, E. Arimondo, and O. Morsch, Full Counting Statistics and Phase Diagram of a Dissipative Rydberg Gas, Phys. Rev. Lett. 113, 023006 (2014).

[31] A. Urvoy, F. Ripka, I. Lesanovsky, D. Booth, J. P. Shaffer, T. Pfau, and R. Löw, Strongly Correlated Growth of Rydberg
Aggregates in a Vapor Cell, Phys. Rev. Lett. 114, 203002 (2015).

[32] M. Ebert, M. Kwon, T. G. Walker, and M. Saffman, Coherence and Rydberg Blockade of Atomic Ensemble Qubits, Phys. Rev. Lett. 115, 093601 (2015).

[33] R. Faoro, C. Simonelli, M. Archimi, G. Masella, M. M. Valado, E. Arimondo, R. Mannella, D. Ciampini, and O. Morsch, van der Waals Explosion of Cold Rydberg Clusters, Phys. Rev. A 93, 030701 (2016).

[34] M. Saffman, T. G. Walker, and K. Mølmer, Quantum Information with Rydberg Atoms, Rev. Mod. Phys. 82, 2313 (2010).

[35] M. Fleischhauer, A. Imamoglu, and J. P. Marangos, Electromagnetically Induced Transparency: Optics in Coherent Media, Rev. Mod. Phys. 77, 633 (2005).

[36] I. Friedler, D. Petrosyan, M. Fleischhauer, and G. Kurizki, Long-Range Interactions and Entanglement of Slow SinglePhoton Pulses, Phys. Rev. A 72, 043803 (2005).

[37] J. D. Pritchard, D. Maxwell, A. Gauguet, K. J. Weatherill, M. P. A. Jones, and C. S. Adams, Cooperative Atom-Light Interaction in a Blockaded Rydberg Ensemble, Phys. Rev. Lett. 105, 193603 (2010).

[38] H. Schempp, G. Günter, C. S. Hofmann, C. Giese, S. D. Saliba, B. D. DePaola, T. Amthor, M. Weidemüller, S. Sevinçli, and T. Pohl, Coherent Population Trapping with Controlled Interparticle Interactions, Phys. Rev. Lett. 104, 173602 (2010).

[39] C. Ates, S. Sevinçli, and T. Pohl, Electromagnetically Induced Transparency in Strongly Interacting Rydberg Gases, Phys. Rev. A 83, 041802 (2011).

[40] S. Sevinçli, N. Henkel, C. Ates, and T. Pohl, Nonlocal Nonlinear Optics in Cold Rydberg Gases, Phys. Rev. Lett. 107, 153001 (2011).

[41] D. Petrosyan, J. Otterbach, and M. Fleischhauer, Electromagnetically Induced Transparency with Rydberg Atoms, Phys. Rev. Lett. 107, 213601 (2011).

[42] A. V. Gorshkov, J. Otterbach, M. Fleischhauer, T. Pohl, and M. D. Lukin, Photon-Photon Interactions via Rydberg Blockade, Phys. Rev. Lett. 107, 133602 (2011).

[43] A. V. Gorshkov, R. Nath, and T. Pohl, Dissipative ManyBody Quantum Optics in Rydberg Media, Phys. Rev. Lett. 110, 153601 (2013).

[44] D. Petrosyan, Dipolar Exchange Induced Transparency with Rydberg Atoms, New J. Phys. 19, 033001 (2017).

[45] S. Das, A. Grankin, I. Iakoupov, E. Brion, J. Borregaard, R. Boddeda, I. Usmani, A. Ourjoumtsev, P. Grangier, and A. S. Sørensen, Photonic Controlled-Phase Gates through Rydberg Blockade in Optical Cavities, Phys. Rev. A 93, 040303 (2016).

[46] M. Fleischhauer and M. D. Lukin, Dark-State Polaritons in Electromagnetically Induced Transparency, Phys. Rev. Lett. 84, 5094 (2000).

[47] M. Fleischhauer and M. D. Lukin, Quantum Memory for Photons: Dark-State Polaritons, Phys. Rev. A 65, 022314 (2002).

[48] Y. O. Dudin and A. Kuzmich, Strongly Interacting Rydberg Excitations of a Cold Atomic Gas, Science 336, 887 (2012).

[49] T. Peyronel, O. Firstenberg, Q.-Y. Liang, S. Hofferberth, A. V. Gorshkov, T. Pohl, M. D. Lukin, and V. Vuletić, Quantum Nonlinear Optics with Single Photons Enabled 
by Strongly Interacting Atoms, Nature (London) 488, 57 (2012).

[50] O. Firstenberg, T. Peyronel, Q.-Y. Liang, A. V. Gorshkov, M. D. Lukin, and V. Vuletić, Attractive Photons in a Quantum Nonlinear Medium, Nature (London) 502, 71 (2013).

[51] V. Parigi, E. Bimbard, J. Stanojevic, A. J. Hilliard, F. Nogrette, R. Tualle-Brouri, A. Ourjoumtsev, and P. Grangier, Observation and Measurement of Interaction-Induced Dispersive Optical Nonlinearities in an Ensemble of Cold Rydberg Atoms, Phys. Rev. Lett. 109, 233602 (2012).

[52] D. Maxwell, D. J. Szwer, D. Paredes-Barato, H. Busche, J. D. Pritchard, A. Gauguet, K. J. Weatherill, M. P. A. Jones, and C. S. Adams, Storage and Control of Optical Photons Using Rydberg Polaritons, Phys. Rev. Lett. 110, 103001 (2013).

[53] D. Maxwell, D. J. Szwer, D. Paredes-Barato, H. Busche, J. D. Pritchard, A. Gauguet, M. P. A. Jones, and C. S. Adams, Microwave Control of the Interaction between Two Optical Photons, Phys. Rev. A 89, 043827 (2014).

[54] S. Baur, D. Tiarks, G. Rempe, and S. Dürr, Single-Photon Switch Based on Rydberg Blockade, Phys. Rev. Lett. 112, 073901 (2014).

[55] D. Tiarks, S. Baur, K. Schneider, S. Dürr, and G. Rempe, Single-Photon Transistor Using a Förster Resonance, Phys. Rev. Lett. 113, 053602 (2014).

[56] H. Gorniaczyk, C. Tresp, J. Schmidt, H. Fedder, and S. Hofferberth, Single-Photon Transistor Mediated by Interstate Rydberg Interactions, Phys. Rev. Lett. 113, 053601 (2014).

[57] H. Gorniaczyk, C. Tresp, P. Bienias, A. Paris-Mandoki, W. Li, I. Mirgorodskiy, H. P. Büchler, I. Lesanovsky, and S. Hofferberth, Enhancement of Rydberg-Mediated SinglePhoton Nonlinearities by Electrically Tuned Förster Resonances, Nat. Commun. 7, 12480 (2016).

[58] D. Tiarks, S. Schmidt, G. Rempe, and S. Durr, Optical Phase Shift Created with a Single-Photon Pulse, Sci. Adv. 2, e1600036 (2016).

[59] C. Tresp, C. Zimmer, I. Mirgorodskiy, H. Gorniaczyk, A. Paris-Mandoki, and S. Hofferberth, Single-Photon Absorber Based on Strongly Interacting Rydberg Atoms, Phys. Rev. Lett. 117, 223001 (2016).

[60] G. Günter, M. Robert-de-Saint-Vincent, H. Schempp, C. S. Hofmann, S. Whitlock, and M. Weidemüller, Interaction Enhanced Imaging of Individual Rydberg Atoms in Dense Gases, Phys. Rev. Lett. 108, 013002 (2012).

[61] G. Günter, H. Schempp, M. Robert-de-Saint-Vincent, V. Gavryusev, S. Helmrich, C. S. Hofmann, S. Whitlock, and M. Weidemüller, Observing the Dynamics of Dipole-Mediated Energy Transport by Interaction-Enhanced Imaging, Science 342, 954 (2013).

[62] C. R. Murray, A. R. Gorshkov, and T. Pohl, Many-Body Decoherence Dynamics and Optimized Operation of a Single-Photon Switch, New J. Phys. 18, 092001 (2016).

[63] E. Zeuthen, M. J. Gullans, M. F. Maghrebi, and A. V. Gorshkov, Correlated Photon Dynamics in Dissipative Rydberg Media, arXiv:1608.06068 [Phys. Rev. Lett. (to be published)].

[64] A. Gaj, A. T. Krupp, J. B. Balewski, R. Löw, S. Hofferberth, and T. Pfau, From Molecular Spectra to a Density Shift in Dense Rydberg Gases, Nat. Commun. 5, 4546 (2014).
[65] M. D. Lukin, M. Fleischhauer, R. Cote, L. M. Duan, D. Jaksch, J. I. Cirac, and P. Zoller, Dipole Blockade and Quantum Information Processing in Mesoscopic Atomic Ensembles, Phys. Rev. Lett. 87, 037901 (2001).

[66] S. E. Harris, J. E. Field, and A. Kasapi, Dispersive Properties of Electromagnetically Induced Transparency, Phys. Rev. A 46, R29 (1992).

[67] A. André and M. D. Lukin, Manipulating Light Pulses via Dynamically Controlled Photonic Band Gap, Phys. Rev. Lett. 89, 143602 (2002).

[68] A. André, M. Bajcsy, A. S. Zibrov, and M. D. Lukin, Nonlinear Optics with Stationary Pulses of Light, Phys. Rev. Lett. 94, 063902 (2005).

[69] M. Fleischhauer, J. Otterbach, and R. G. Unanyan, BoseEinstein Condensation of Stationary-Light Polaritons, Phys. Rev. Lett. 101, 163601 (2008).

[70] F. E. Zimmer, J. Otterbach, R. G. Unanyan, B. W. Shore, and M. Fleischhauer, Dark-State Polaritons for Multicomponent and Stationary Light Fields, Phys. Rev. A 77, 063823 (2008).

[71] M. Hafezi, D. E. Chang, V. Gritsev, E. Demler, and M. D. Lukin, Quantum Transport of Strongly Interacting Photons in a One-Dimensional Nonlinear Waveguide, Phys. Rev. A 85, 013822 (2012).

[72] I. Iakoupov, J. R. Ott, D. E. Chang, and A.S. Sørensen, Dispersion Relations for Stationary Light in One-Dimensional Atomic Ensembles, Phys. Rev. A 94, 053824 (2016).

[73] L. Vestergaard Hau, S. E. Harris, Z. Dutton, and C. H. Behroozi, Light Speed Reduction to 17 Metres per Second in an Ultracold Atomic Gas, Nature (London) 397, 594 (1999).

[74] M. M. Kash, V. A. Sautenkov, A. S. Zibrov, L. Hollberg, G. R. Welch, M. D. Lukin, Y. Rostovtsev, E. S. Fry, and M. O. Scully, Ultraslow Group Velocity and Enhanced Nonlinear Optical Effects in a Coherently Driven Hot Atomic Gas, Phys. Rev. Lett. 82, 5229 (1999).

[75] M. Bajcsy, A. S. Zibrov, and M. D. Lukin, Stationary Pulses of Light in an Atomic Medium, Nature (London) 426, 638 (2003).

[76] Y.-W. Lin, W.-T. Liao, T. Peters, H.-C. Chou, J.-S. Wang, H.-W. Cho, P.-C. Kuan, and I. A. Yu, Stationary Light Pulses in Cold Atomic Media and without Bragg Gratings, Phys. Rev. Lett. 102, 213601 (2009).

[77] J. L. Everett, G. T. Campbell, Y.-W. Cho, P. Vernaz-Gris, D. B. Higginbottom, O. Pinel, N. P. Robins, P. K. Lam, and B. C. Buchler, Dynamical Observations of Self-Stabilizing Stationary Light, Nat. Phys. 13, 68 (2017).

[78] I. Novikova, A. V. Gorshkov, D. F. Phillips, A. S. Sørensen, M. D. Lukin, and R. L. Walsworth, Optimal Control of Light Pulse Storage and Retrieval, Phys. Rev. Lett. 98, 243602 (2007).

[79] A. V. Gorshkov, A. André, M. Fleischhauer, A. S. Sørensen, and M. D. Lukin, Universal Approach to Optimal Photon Storage in Atomic Media, Phys. Rev. Lett. 98, 123601 (2007).

[80] A. V. Gorshkov, A. André, M. D. Lukin, and A. S. Sørensen, Photon Storage in $\Lambda$-Type Optically Dense Atomic Media. II. Free-Space Model, Phys. Rev. A 76, 033805 (2007).

[81] W. Li and I. Lesanovsky, Coherence in a Cold-Atom Photon Switch, Phys. Rev. A 92, 043828 (2015). 
[82] K. Xia and J. Twamley, All-Optical Switching and Router via the Direct Quantum Control of Coupling between Cavity Modes, Phys. Rev. X 3, 031013 (2013).

[83] D. E. Chang, A. S. Sørensen, E. A. Demler, and M. D. Lukin, A Single-Photon Transistor Using Nanoscale Surface Plasmons, Nat. Phys. 3, 807 (2007).

[84] M. Bajcsy, S. Hofferberth, V. Balic, T. Peyronel, M. Hafezi, A. S. Zibrov, V. Vuletic, and M. D. Lukin, Efficient AllOptical Switching Using Slow Light within a Hollow Fiber, Phys. Rev. Lett. 102, 203902 (2009).

[85] K. Nozaki, T. Tanabe, A. Shinya, S. Matsuo, T. Sato, H. Taniyama, and M. Notomi, Sub-Femtojoule All-Optical Switching Using a Photonic-Crystal Nanocavity, Nat. Photonics 4, 477 (2010).

[86] T. Volz, A. Reinhard, M. Winger, A. Badolato, K. J. Hennessy, E. L. Hu, and A. Imamolu, Ultrafast All-Optical Switching by Single Photons, Nat. Photonics 6, 607 (2012).

[87] R. Bose, D. Sridharan, H. Kim, G. S. Solomon, and E. Waks, Low-Photon-Number Optical Switching with a Single Quantum Dot Coupled to a Photonic Crystal Cavity, Phys. Rev. Lett. 108, 227402 (2012).

[88] W. Chen, K. M. Beck, R. Bücker, M. Gullans, M. D. Lukin, H. Tanji-Suzuki, and V. Vuletić, All-Optical Switch and Transistor Gated by One Stored Photon, Science 341, 768 (2013).

[89] D. O'Shea, C. Junge, J. Volz, and A. Rauschenbeutel, FiberOptical Switch Controlled by a Single Atom, Phys. Rev. Lett. 111, 193601 (2013).
[90] Y.-H. Chen, M.-J. Lee, W. Hung, Y.-C. Chen, Y.-F. Chen, and I. A. Yu, Demonstration of the Interaction between Two Stopped Light Pulses, Phys. Rev. Lett. 108, 173603 (2012).

[91] T. Peters, S.-W. Su, Y.-H. Chen, J.-S. Wang, S.-C. Gou, and I. A. Yu, Formation of Stationary Light in a Medium of Nonstationary Atoms, Phys. Rev. A 85, 023838 (2012).

[92] J. D. Thompson, T. L. Nicholson, Q.-Y. Liang, S. H. Cantu, A. V. Venkatramani, S. Choi, I. A. Fedorov, D. Viscor, T. Pohl, M. D. Lukin, and V. Vuletić, Symmetry-Protected Collisions between Strongly Interacting Photons, Nature (London) 542, 206 (2017).

[93] K. Singer, J. Stanojevic, M. Weidemüller, and R. Côté, Long-Range Interactions between Alkali Rydberg Atom Pairs Correlated to the $n \mathrm{~s}-n \mathrm{~s}, n \mathrm{p}-n \mathrm{p}$ and $n \mathrm{~d}-n \mathrm{~d}$ Asymptotes, J. Phys. B 38, S295 (2005).

[94] J. Petersen, J. Volz, and A. Rauschenbeutel, Chiral Nanophotonic Waveguide Interface Based on Spin-Orbit Interaction of Light, Science 346, 67 (2014).

[95] R. Mitsch, C. Sayrin, B. Albrecht, P. Schneeweiss, and A. Rauschenbeutel, Quantum State-Controlled Directional Spontaneous Emission of Photons into a Nanophotonic Waveguide, Nat. Commun. 5, 5713 (2014).

[96] H. Pichler, T. Ramos, A. J. Daley, and P. Zoller, Quantum Optics of Chiral Spin Networks, Phys. Rev. A 91, 042116 (2015).

[97] P. Lodahl, S. Mahmoodian, S. Stobbe, A. Rauschenbeutel, P. Schneeweiss, J. Volz, H. Pichler, and P. Zoller, Chiral Quantum Optics, Nature (London) 541, 473 (2017). 